\title{
Évolution, modélisation et cartographie des rendements de l'oliveraie dans la province de Jaen en Espagne (1959-2018)
}

\author{
Fatma Arfaoui ${ }^{1}$, Marianne Cohen ${ }^{2}$, Ludovic Oudin ${ }^{3}$ et Josyane Ronchail ${ }^{4 *}$ \\ ${ }^{1}$ Sorbonne Université, Paris, France \\ ${ }^{2}$ Laboratoire Médiations, Faculté des Lettres, Sorbonne Université, Paris, France \\ ${ }^{3}$ Sorbonne Université, CNRS, EPHE, UMR METIS, Case 105, 4 Place Jussieu, F-75005 Paris, France \\ ${ }^{4}$ Laboratoire d'Océanographie et du Climat, LOCEAN-IPSL, Sorbonne Université, IRD, CNRS, MNHN, Paris, France
}

\begin{abstract}
Résumé - Les acteurs de l'oléiculture en Andalousie s'interrogent sur l'impact du changement climatique sur leur activité, très importante en termes de surfaces concernées, de production et de revenus. Pour tenter de leur répondre, ce travail décrit tout d'abord l'évolution du climat et des rendements annuels des oliveraies pluviales et irriguées dans la province de Jaén (Andalousie) depuis les années 50. Puis sont réalisées des modélisations des relations entre les rendements pluviaux et irrigués provinciaux et des variables climatiques TerraClimate (à résolution de $4 \mathrm{~km}$ ), moyennées sur la zone oléicole de la province de Jaén. Enfin, les modèles permettent de calculer puis de cartographier la répartition théorique actuelle des rendements. Les rendements, très variables d'une année sur l'autre, plus élevés dans les vergers irrigués que dans les vergers pluviaux, ont fortement augmenté depuis la fin des années cinquante puis ils se sont stabilisés à partir de la fin des années 1990, avec en moyenne $30 \mathrm{q} / \mathrm{ha}$ en pluvial et $51 \mathrm{q} / \mathrm{ha}$ en irrigué pour la période 1996-2018. Par ailleurs, les températures, moyennées sur la zone oléicole de la province de Jaén, ont augmenté, surtout les températures diurnes, essentiellement durant la saison chaude. Les précipitations, très variables d'une année sur l'autre, ont diminué de $16 \%$ après 1979. Entre 1996 et 2018, les variables qui expliquent le mieux les rendements sont les pluies annuelles et les températures minimales d'automne et d'août dans les oliveraies pluviales, et les pluies et températures minimales d'automne, les températures maximales d'été et les températures minimales de février, dans les oliveraies irriguées. Des valeurs de rendement théoriques en pluvial et en irrigué sont calculées par régression multiple (1996-2018), validées par cross-validation. Les valeurs théoriques sont ensuite cartographiées sur des pixels de $4 \mathrm{~km}$ de côté. Si les valeurs simulées et moyennées (1996-2018) sont proches des valeurs provinciales observées, avec une erreur-moyenne de $6 \mathrm{q} / \mathrm{ha}$ en irrigué et en pluvial, leur répartition spatiale n'est pas tout à fait cohérente avec quelques informations disponibles à l'échelle communale. Il conviendra donc de perfectionner la modélisation des rendements et leur cartographie avant de passer à l'étape suivante : simuler et cartographier les rendements potentiels futurs, compte tenu du changement climatique, pour disposer d'un outil de discussion avec les oléiculteurs et les aider à préparer l'adaptation de leur activité.
\end{abstract}

Mots-clés : climat, rendement, oliveraie, Andalousie, province de Jaén, cartographie.

\begin{abstract}
Evolution, modeling and mapping of olive grove yields in the province of Jaen, Spain (1959-2018). Olive growers in Andalusia are concerned about the impact of climate change on their activity, which is very important in terms of surface area, production and income. In an attempt to answer these questions, this work first describes the evolution of climate and annual yields of rainfed and irrigated olive groves in the province of Jaén (Andalusia) since the 1950s. Second, the relationships between rainfed and irrigated yields on one side and climatic variables on the other side are modelled. Finally, the models allow the calculation and mapping of the current theoretical spatial distribution of yields. Yields vary greatly from year to year, but are generally higher in irrigated than in rainfed orchards. Yields have risen sharply since the end of the 1950s, stabilizing since the end of the 1990s, with an average over the 1996-2018 period of $30 \mathrm{q} / \mathrm{ha}$ and $51 \mathrm{q} / \mathrm{ha}$ in irrigated and rainfed areas, respectively. Meanwhile, the mean temperatures over the olive growing area of the province of Jaén, have increased, especially for daytime and during the hot season. Rainfall, which is highly variable over the studied area, has decreased by 16\% after 1979. Between 1996 and 2018, the climatic variables explaining best olive yields for rainfed areas are annual rainfall and minimum temperatures in autumn and August. Concerning irrigated areas, autumn rainfall and minimum temperatures, maximum summer temperatures and minimum
\end{abstract}


February temperatures are the most relevant climatic variables. Theoretical yield values for rainfed and irrigated olive groves are calculated by multiple regression (1996-2018), validated by cross-validation. Theoretical yield values are then mapped on $4 \mathrm{~km}$ side pixels. Although the simulated and averaged values (1996-2018) are close to the observed provincial values, with a mean error of $6 \mathrm{q} / \mathrm{ha}$ in irrigated and rainfed areas, their spatial distribution is not entirely consistent with some information available at the communal level. It will therefore be necessary to improve the modeling of yields and their mapping before moving on to the next step: simulating and mapping potential future yields, taking into account climate change, in order to have a tool for discussion with olive growers and to help them prepare the adaptation of their activity.

Keywords: climate, yield, olive groves, Andalucía, Jaen province, mapping.

* $\overline{\text { Auteure de correspondance }}$ : josyane.ronchail@locean.ipsl.fr

\section{Introduction}

Le changement climatique a des conséquences particulièrement importantes dans la région méditerranéenne (IPCC, 2014; Lionello et Scarascia, 2018 ; MedECC, 2020 ; Ranasinghe et al., 2021), et affecte les rendements agricoles, avec des conséquences socio-économiques importantes dans les régions de monoculture (PNUE/PAM et Plan Bleu, 2020). C'est le cas de l'oléiculture dans la Province de Jaén. Malgré les progrès agronomiques accomplis depuis les années 50, et le développement de l'irrigation à partir des années 90 (Araque et al., 2002), l'augmentation des températures due au changement climatique modifie le cycle phénologique (Gabaldón-Leal et al., 2017), la diminution des pluies accentue le stress hydrique et limite les ressources hydriques nécessaires pour l'irrigation (Tanasijevic et al., 2014), l'abondance des larves de la mouche de l'olivier augmente (Ponti et al., 2014), toutes choses qui affectent les rendements et fragilisent encore plus l'environnement (Sánchez-Martinez et PanizaCabrera, 2015) et l'économie locale (SánchezMartínez et Garrido-Almonacid, 2017). En effet, bien que l'olivier soit très résistant à la sécheresse car il pousse avec moins de $200 \mathrm{~mm}$ dans des vergers très peu denses (Baldy, 1990; Angles, 2016), celle-ci affecte les rendements qui sont la clé de la durabilité économique de cette culture. Dans le cadre du programme de recherche OLICLIM, un partenariat est développé avec les acteurs de la filière oléicole et les aménageurs, afin de coconstruire des stratégies de transformation de l'oliveraie en une « oliveraie intelligente » face au changement climatique.

Etablir une carte des rendements théoriques actuels de l'oléiculture dans la région de Jaén permet d'apporter de nouvelles informations sur lesquelles les acteurs peuvent s'appuyer pour poursuivre le dialogue sur l'adaptation au changement climatique. Il s'agit d'un préalable à la construction de cartes de rendements potentiels futurs, pour localiser les pertes les plus importantes et susciter des réflexions sur les stratégies d'implantation de futures oliveraies. Cette connaissance permettra d'anticiper des actions, ce qui est particulièrement important pour l'arboriculture. Ces recherches font suite à de nombreux travaux de collègues des pays du pourtour méditerranéen qui cherchent à modéliser les rendements futurs des oliveraies et à développer des stratégies d'adaptation aussi bien pour l'oliveraie (Fraga et al., 2021 ; Arenas-Castro et al., 2020, entre autres) que pour d'autres cultures emblématiques comme la vigne (van Leeuven et al., 2019). Ces derniers travaux se distinguent notamment par l'utilisation de données à échelle très fine (Quenol et Bonnardot, 2014) et une approche de modélisation multi-agent qui permet de représenter aussi bien le climat local et le cycle physiologique de la plante que les stratégies des acteurs de la production viticole (Tissot et al., 2020).

Cet article a pour objectif d'exposer la démarche suivie pour mettre en place la cartographie des rendements théoriques des oliveraies dans la province de Jaén. Dans un premier temps, sont présentés les évolutions des rendements, des précipitations et des températures moyennés à l'échelle de l'ensemble des surfaces oléicoles de la province de Jaén, puis les modèles statistiques qui mettent en relation climat et rendements en pluvial et en irrigué à cette même échelle. Ces modèles 
permettent grâce à la disponibilité de données climatiques à relativement haute résolution spatiale $(\sim 4 \mathrm{~km})$ de simuler des rendements théoriques et de les cartographier. Les cartes résultantes sont présentées et leur validité et leur intérêt pour la suite de notre recherche sont discutés.

\section{Présentation de l'étude}

Dans cette partie sont décrites la région d'étude et les enjeux économiques autour de l'olivier, les données agricoles et climatiques utilisées ainsi que les méthodes mises en œuvre.

\subsection{L'oliveraie dans la province de Jaén}

La province de Jaén (13 $489 \mathrm{~km}^{2}$ ) est située en Andalousie, à $37^{\circ} \mathrm{N}$, dans la haute vallée du Guadalquivir (figure 1); $5900 \mathrm{~km}^{2}$ sont couverts d'oliveraies en 2018, ce qui représente un tiers des oliveraies andalouses et un cinquième des oliveraies espagnoles (MAPA, 2021a). Ainsi, dans la province de Jaén, les oliveraies couvrent $90 \%$ de la surface agricole utile et permettent de produire davantage d'huile d'olive (470 000 t par an, moyenne 20032012) que la Grèce et l'Italie réunies (SanchezMartinez et Paniza-Cabrera, 2015 ; RodríguezCohard et al., 2017). L'économie de la province dépend donc fortement de la monoculture de l'olivier (Sánchez-Martínez et Paniza-Cabrera, 2015 ; Sánchez-Martínez et al., 2020).

Dans cette région, les oliveraies occupent les vallées et les bas de versants, alors que les chaines de montagne bordant la Vallée du Guadalquivir et culminant à plus de $2000 \mathrm{~m}$ sont couvertes de surfaces forestières et de rochers à leurs sommets (figures 1 et $2 \mathrm{a}$ ). En effet, même si l'olivier est considéré comme l'une des espèces les plus appropriées et les mieux adaptées au climat méditerranéen (Sofo et al., 2008 ; Angles, 2016) aux étés longs, chauds et secs, et aux hivers doux et humides, des limites climatiques lui sont imposées. L'olivier est sensible au gel hivernal, les feuilles commençant à geler après une exposition de quelques heures à $-5^{\circ} \mathrm{C}$, ce qui fait qu'il est essentiellement présent en deçà de $1000 \mathrm{~m}$ d'altitude dans la province de Jaén. La période d'induction florale jusqu'à la floraison nécessite des températures basses $\left(7^{\circ} \mathrm{C}\right.$ en indice actinométrique) mais les températures ne doivent pas dépasser $40^{\circ} \mathrm{C}$ en été (Baldy, 1990). De fait, la variabilité du climat et la physiologie de l'arbre ont une influence importante sur les rendements de l'olivier dans cette province. Cohen et al. (2014) mettent en évidence l'importance du cumul des pluies des deux années passées dans leurs modélisations des rendements en pluvial et en irrigué dans la province de Jaén et Bades-Maquet (2018) introduit les températures de fin d'hiver et celles de fin de printemps dans les siennes. Galan et al. (2008) ou Moriondo et al. (2015) soulignent l'importance des pluies de printemps et de fin d'été et les températures d'hiver et d'été. Arenas-Castro et al. (2020) insistent sur les pluies d'été et d'automne, ce qui correspond aux indications données par les oléiculteurs (Cohen et al., 2014).

Les oliveraies sont en grande partie irriguées, au printemps et en été : $41 \%$ des surfaces oléicoles de la province de Jaén le sont en 2018, essentiellement dans la partie centrale et basse de la province de Jaén (comarque de la Loma), le long du cours du Guadalquivir et dans les basses vallées de ses affluents (figure 2a). La dotation actuelle, de 1500 $\mathrm{m}^{3} / \mathrm{ha}$, a baissé de moitié depuis 1995 et est légèrement supérieure aux recommandations des autorités de bassin $\left(1290 \mathrm{~m}^{3} / \mathrm{ha}\right.$; Cohen et al., 2014) mais représente un acquis important pour les communautés d'irrigants (enquêtes de 2018-2019). Les surfaces irriguées n'ont cessé de progresser depuis l'entrée de l'Espagne dans l'Union Européenne (1986) aux dépens des surfaces pluviales et d'autres cultures (Araque et al., 2002 ; figure 2a). Néanmoins, de vastes régions de plaine et de piedmont comme la Comarque de la Campiña de Jaén, à l'ouest, portent essentiellement des oliveraies pluviales. Plus généralement, l'extension des oliveraies pluviales et irriguées aux dépens des autres cultures s'est faite depuis la partie centrale de la province, le long du Guadalquivir, vers l'est et les montagnes du sud, à l'exception des régions les plus élevées (figure 2b). 


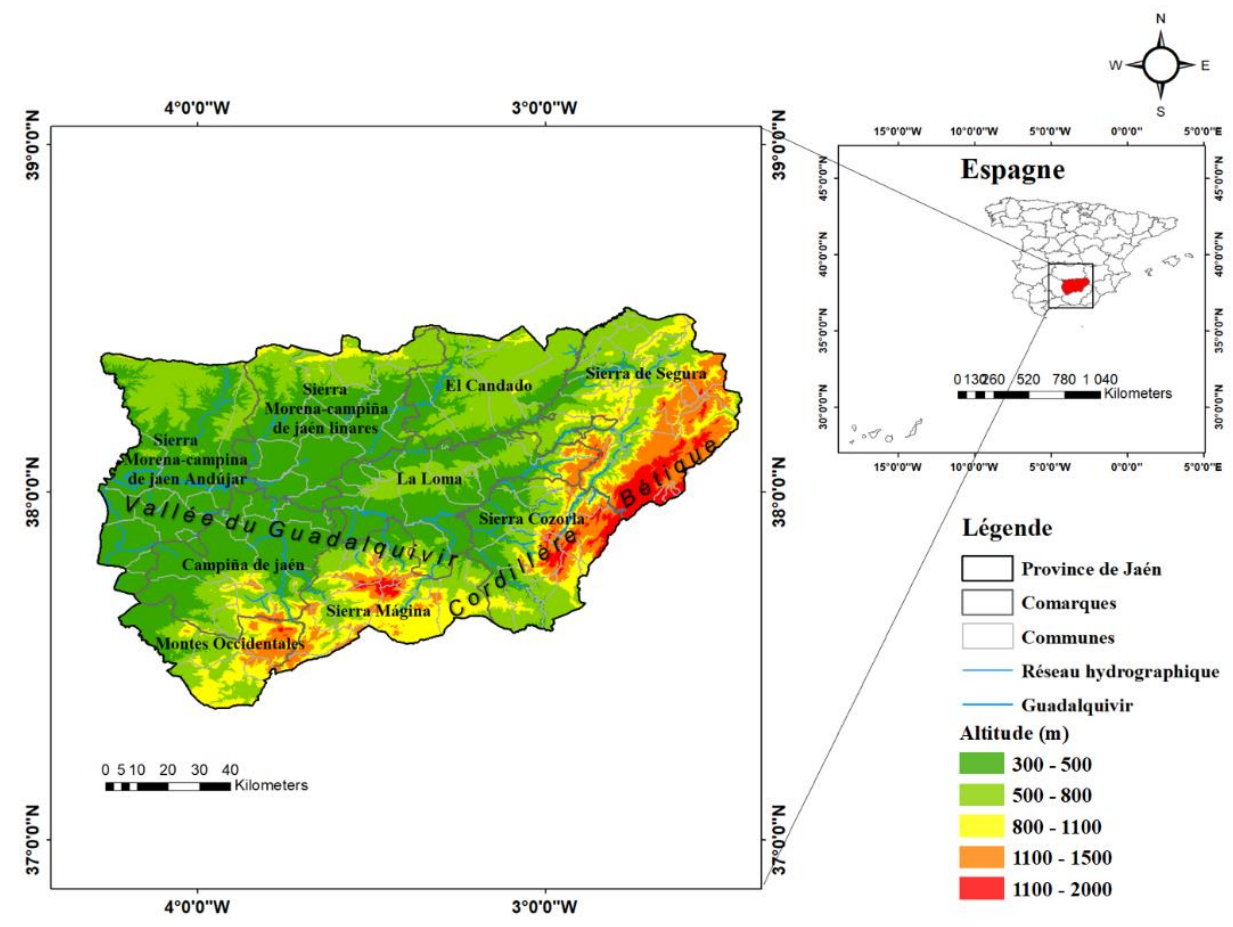

Figure 1. La province de Jaén, relief, hydrographie et limites des comarques qui subdivisent la province de Jaén en 9 sous-unités administratives. The province of Jaén, relief, hydrography and boundaries of the counties that subdivide the province of Jaén into 9 administrative sub-units.

Figure 2. a) Extension des oliveraies pluviales et irriguées dans la province de Jaén (source des données : SIGPAC \& MAGRAMA, 2018); b) Evolution des surfaces oléicoles pluviales et irriguées 1959-2018 (MAPA, 2021b). a) Extension of rainfed and irrigated olive groves in the province of Jaén (source of the data: SIGPAC \& MAGRAMA, 2018), b) Evolution of rainfed and irrigated olive growing areas 19592018 (MAPA 2021b).
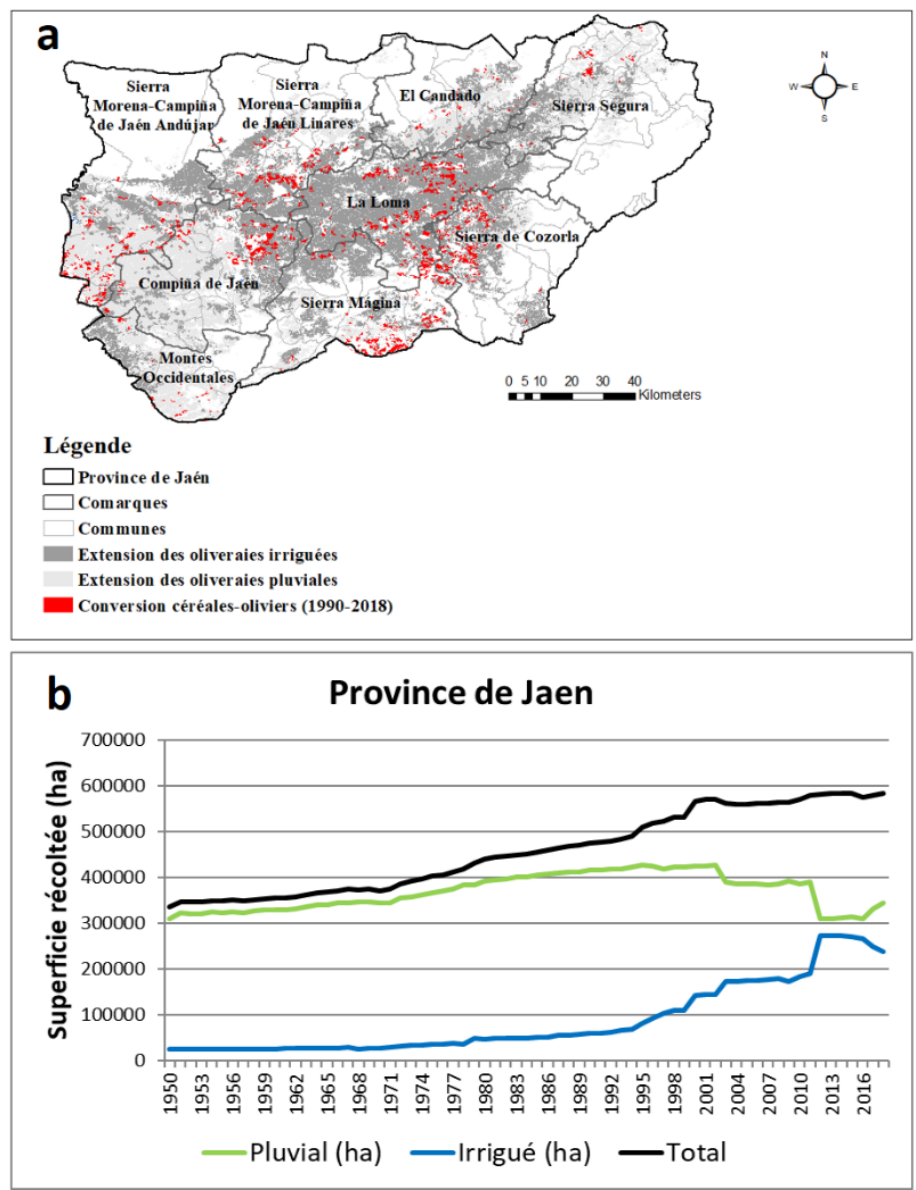


\subsection{Données}

Les données de rendements annuelles des oliveraies pluviales et irriguées (en quintaux par hectare) sont disponibles à l'échelle provinciale, sur le site web du ministère de l'agriculture, de la pêche et de l'alimentation (MAPA, 2021b) pour la période 1959-2018. Des données de production et de surface, à l'échelle communale, sont mises à disposition par la Junta de Andalucia (OCA, 2001). Elles sont utilisées pour une validation spatiale des résultats de la simulation des rendements à l'échelle de $4 \mathrm{~km}^{2}$ (celle des données climatiques). Mais elles ne sont disponibles que pour 1996 et 1997.

Pour visualiser l'évolution de l'utilisation des sols au sein de la province de Jaén, nous avons utilisé la carte des cultures et de l'utilisation des sols au 1:50 $000^{\text {ème }}$ disponible en format numérique à deux périodes : 1980-1990 et 2000-2010 (GeoPortal du MAPAMA, 2021, sig.mapama.gob.es/geoportal/). Les cartes diachroniques Corine Land Cover (CLC, 2021, land.copernicus.eu/pan-european/corine-landcover/) montrent les changements d'affectation de la céréaliculture vers l'oléiculture entre millésimes successifs (1990, 2000, 2006, 2012 et 2018), à partir des données collectées par le programme européen d'observation de la terre Copernicus. La figure $2 b$ résulte d'une fusion des polygones correspondant à un changement de la céréaliculture vers l'oléiculture pour les périodes 1990-2000, 2000-2006, 2006-2012 et 2012-2018.

Bien qu'elles ne soient pas utilisées dans l'analyse, mais plutôt pour l'éclairer ou dans la discussion, nous mentionnons les données d'enquêtes, réalisées entre 2009 et 2019 dans la Comarque de Sierra Mágina, auprès de professionnels de l'oléiculture et de l'aménagement (84 entretiens individuels et ateliers) et d'habitants (une centaine de questionnaires). Ces enquêtes ont permis de documenter les systèmes agricoles, les pratiques d'irrigation ainsi que les connaissances des agriculteurs sur les facteurs climatiques (et autres) du rendement des oliviers. Mentionnons aussi nos échanges avec les géographes de l'Université de Jaén ou y ayant résidé, cités dans cet article, et qui nous ont guidé dans la compréhension de cette région oléicole.
Les données climatiques proviennent de TerraClimate. Il s'agit d'une réanalyse à l'échelle du globe d'une résolution spatiale $1 / 24^{\grave{e ̀ m e}}$ de degré, soit environ $4 \mathrm{~km}$ sur la zone d'étude, au pas de temps mensuel qui combine plusieurs réanalyses climatiques (Abatzoglou et al., 2018) : WorldClim v1.4 et v2 (Fick et Hijmans, 2017 ; Hijmans et al., 2005), CRU Ts4.0 (Harris et al., 2020), et JRA-55 (Kobayashi et al., 2015). La compilation des données originales se fait en plusieurs étapes. Dans un premier temps, des moyennes climatiques mensuelles à haute résolution sont dérivées de WorldClim v2. Des séries temporelles d'anomalies climatiques avec des produits à plus faible résolution spatiale (CRU Ts4.0 et JRA-55) sont ensuite utilisées pour constituer les séries de données TerraClimate, en superposant les anomalies climatiques mensuelles de l'étape 3 aux normales climatiques mensuelles.

Cette opération est réalisée à l'aide d'une interpolation climatologiquement assistée consistant en une simple réduction d'échelle spatiale avec interpolation bilinéaire des anomalies temporelles (Mosier et al., 2014 ; Willmott et Robeson, 1995).

Des valeurs mensuelles de pluie (en $\mathrm{mm}$ ) et de températures maximales et minimales $\left(\mathrm{en}{ }^{\circ} \mathrm{C}\right)$ ont été extraites sur les zones cultivées en oliviers dans la province de Jaén pour chaque point de grille avant d'être moyennées. A partir de ces données, nous avons construit un jeu de 71 variables thermiques et pluviométriques à différents pas de temps. Ce sont les suivantes, sachant qu'un cycle de production d'olives commence en septembre de l'année n-1 quand les sols commencent à reconstituer leur réserve en eau et finit en décembre de l'année $\mathrm{n}$, quand s'achève plus ou moins la récolte :

- Pluies, températures minimales et maximales de chaque mois de l'année et de l'automne précédent (de septembre à décembre), soit trois fois 16 variables;

- Pluies, températures minimales et maximales des trimestres correspondant, soit trois fois 5 variables ; 
- Pluies, températures minimales et maximales annuelles, avec des regroupements par année hydrologique (de septembre de l'année n-1 à août de l'année n) et par année physiologique (de septembre de l'année n-1 à décembre de l'année n), soit trois fois deux variables ;

- Pluies annuelles intégrées sur deux et trois années hydrologiques. Le cumul pluriannuel des pluies a montré son efficacité explicative dans les travaux de Cohen et al. (2014) et BadiaCanes Maquet (2018).

Toutes ces variables ont été retenues pour la modélisation des rendements. En effet, lors $\mathrm{d}$ 'ateliers avec les agriculteurs dont le thème portait sur les facteurs climatiques qui favorisent les rendements (en 2018 et 2019), il est apparu qu'il ne convenait pas de sélectionner uniquement les variables citées dans la littérature, nos premiers essais de modélisation ayant par ailleurs montré qu'il ne convenait pas non plus de s'en tenir aux variables signalées par les oléiculteurs (Ronchail et al., 2014), ce pourquoi nous avons élargi les possibilités.

\subsection{Démarche et méthodes d'analyse}

Des corrélations de Bravais Pearson sont utilisées pour analyser l'évolution des rendements en pluvial et en irrigué et du climat (températures et pluies) sur la période 1959-2018 et le test de Pettitt est appliqué afin de détecter des ruptures dans les mêmes séries. Les corrélations et les ruptures sont données comme significatives quand p est au moins inférieur à 0,05 .

Deux modèles climat-rendement sont créés à partir des données de la période 1996-2018, un pour les oliveraies pluviales, l'autre pour les oliveraies irriguées. Les modèles sont établis à partir de données de rendements disponibles pour la province de Jaén et de données climatiques de précipitations, températures maximales et minimales extraites des points de grille correspondant aux surfaces en oliveraies et moyennées. On obtient donc deux modèles rendement-climat pour l'ensemble de la province de Jaén. Le choix de la période retenu est expliqué dans le paragraphe 3.1.
La simulation des rendements en sec et en irrigué à partir de variables climatiques mobilise la régression multiple pas à pas sur la période 19962018. Les modèles sont contrôlés par validation croisée selon la procédure LOOCV (Leave-one-out cross-validation). Cette approche laisse une valeur hors des données d'apprentissage ; ainsi, s'il y a $n$ valeurs dans l'échantillon d'origine, $n-1$ valeurs sont utilisées pour entraîner le modèle et 1 valeur est utilisée pour la validation. Ceci est répété pour toutes les combinaisons possibles. Coefficient de détermination, erreur moyenne absolue (MAE), critère d'information d'Akaike (AIC ; Akaike, 1974) et erreur quadratique moyenne (RMSE) sont utilisés pour tester la qualité des modèles.

En faisant l'hypothèse que le lien entre rendement et climat, valable à l'échelle de la province, vaut à une échelle plus fine, ces modèles sont utilisés pour reconstruire des valeurs de rendements théoriques à l'échelle des pixels climatiques en utilisant les données de TerraClimate. Ces valeurs théoriques calculées sur chaque pixel de la grille climatique, y compris pour les pixels qui ne sont pas actuellement couverts d'oliveraie, sont ensuite cartographiées de façon à simuler la variabilité spatiale des rendements sur des pixels de $4 \mathrm{~km}^{2}$. Des cartes de rendements théoriques sont produites chaque année entre 1996 et 2018 ainsi qu'en moyenne pour la période 19962018.

\section{Résultats}

Après l'analyse de l'évolution des rendements et du climat depuis 1959, deux modèles, un pour l'oliveraie pluviale et l'autre pour l'oliveraie irriguée, ont été mis au point à partir des données annuelles de rendements dans la province de Jaén et de 71 variables pluviométriques et thermiques pour la période 1996-2018. Cette double temporalité permet de contextualiser les résultats obtenus pour la période récente.

\subsection{Evolution des rendements des oliveraies dans la province de Jaén}

Les rendements ont augmenté depuis le milieu du $\mathrm{XX}^{\mathrm{e}}$ siècle grâce à l'amélioration des pratiques 
agricoles et pour satisfaire la demande européenne (Sanchez-Martinez et Paniza-Cabrera, 2015) (figure 3a). Cette amélioration s'est accélérée au milieu des années 90 après 3 années sèches (1993-1995, avec

Figure 3. a) Rendements (quintaux/hectare) des oliveraies pluviales et irriguées dans la province de Jaén (Andalousie) de 1959 à 2018. b) Précipitations $(\mathrm{mm})$, températures $\left({ }^{\circ} \mathrm{C}\right)$ maximales et minimales des années hydrologiques (septembre-octobre) dans les surfaces oléicoles de la province de Jaén de 1959 à 2018. a) Yields (quintals/hectare) of rainfed and irrigated olive groves in the province of Jaén (Andalusia) from 1959 to 2018. b) Precipitation ( $\mathrm{mm}$ ), maximum and minimum temperatures $\left({ }^{\circ} \mathrm{C}\right)$ of the hydrological years (September-October) in olivegrowing areas of the province of Jaén from 1959 to 2018.

Le test de rupture de Pettitt appliqué aux rendements présente ainsi une rupture significative en 1996, après la grande sécheresse. Les rendements en pluvial passent de 17,3 à 30,5 q/ha en moyenne entre les périodes $1959-1995$ et 19962018 et les rendements irrigués de 28,3 à 50,6 q/ha. De plus, avant 1996, les rendements progressent significativement dans les vergers irrigués (de 24,5 à 34,3 q/ha entre les dix premières et dix dernières années de la période 1959-1996), mais pas dans les vergers pluviaux où l'augmentation entre les mêmes périodes n'est pas significative (de 15,3 à 21,9 q/ha). A partir de 1996, les rendements ne connaissent pas d'évolution significative. Comptetenu de la stationnarité des rendements pendant la période de 22 ans 1996-2018, celle-ci est retenue pour la modélisation climat-rendement.

Les rendements varient en outre fortement d'une année sur l'autre, ce qui est lié d'une part à l'alternance physiologique des oliviers qui, une des pluies annuelles inférieures à $400 \mathrm{~mm}$ ) auxquelles les oléiculteurs ont réagi notamment en améliorant les systèmes d'irrigation.
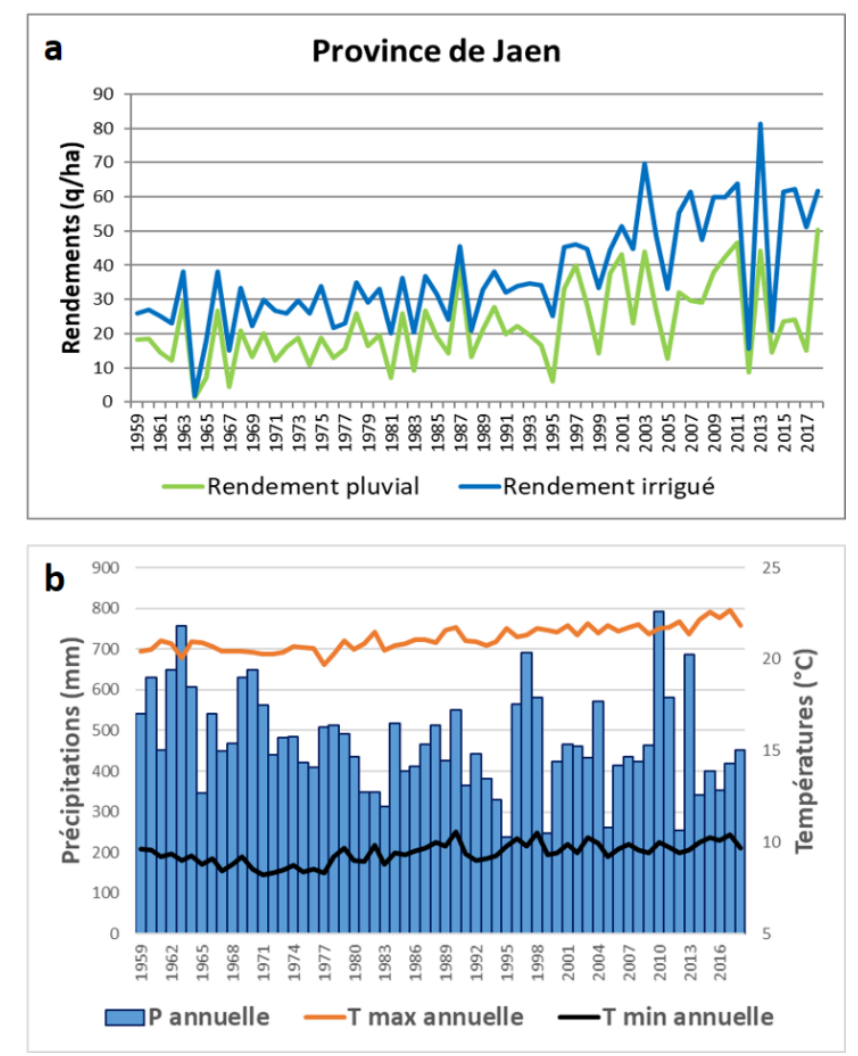

année sur deux en général, mettent en place des rameaux et fructifient l'année suivante et, d'autre part, à la variabilité des précipitations (Cohen et al., 2014).

De plus, il est remarquable et attendu que le rendement moyen des oliveraies irriguées dépasse largement celui des oliveraies pluviales (moyenne de 30,5 q/ha en pluvial et de 50,6 q/ha en irrigué pour la période 1996-2018) et que la variabilité interannuelle des rendements des oliveraies pluviales soit plus accentuée que celle des rendements en irrigué ; en effet, le coefficient de variation est de 0,40 pour le rendement pluvial et de 0,30 en irrigué (période 1996-2018). Les paramètres statistiques montrent par ailleurs que la variabilité interannuelle des rendements ne change pratiquement pas avant et après 1996 ; les coefficients de variation passent de 0,44 à 0,39 pour les rendements en pluvial et de 0,28 à 0,29 pour les rendements des vergers irrigués. 


\subsection{L'évolution des pluies et des températures sur la période 1959-2018 dans les surfaces occupées par l'oliveraie en 2018 dans la province de Jaén}

Pendant la période 1959-2018, les pluies annuelles baissent de $16 \%$ après une rupture significative ( $\mathrm{p}<0,05$, test de Pettitt) en 1979 (de $525 \mathrm{~mm}$ pour la période $1959-1979$ à $440 \mathrm{~mm}$ pour 1980-2018) et leur variabilité augmente de 19 à 28 $\%$ autour de la moyenne après cette date (figure $3 \mathrm{~b}$ ). Ce changement est également observé dans les débits des rivières (Lorenzo-Lacruz et al., 2013 ; Cohen et al., 2014). Pendant la période 1996-2018, retenue pour l'élaboration des modèles de rendements, les pluies annuelles à l'échelle de la province de Jaén sont de $465 \mathrm{~mm} / \mathrm{an}$ et leur variabilité de $30 \%$ autour de la moyenne.

Les températures maximales annuelles augmentent de $1,4^{\circ} \mathrm{C}$ entre la première et la dernière décennie de 1959-2018 (figure 3b). Le test de Pettitt identifie une rupture à la fin des années 80 (en 1988, p <0,01). L'augmentation est particulièrement importante pendant le trimestre estival juin-juillet-août, avec une augmentation de $1,8^{\circ} \mathrm{C}$ entre la dernière et la première décennie de 1959-2018 (non montré). Les températures minimales augmentent aussi au pas de temps annuel $\left(+0,8^{\circ} \mathrm{C}\right.$ entre la dernière et première décennie de la période 1959-2018). Une rupture intervient en 1985 (test de Pettitt $\mathrm{p}<0,01$ ). Au pas de temps mensuel, l'augmentation n'est significative que de mars à octobre (non montré). Les valeurs d'amplitudes thermiques diurnes annuelles, quant à elles, augmentent significativement pendant la période $1959-2018$ : de $11,5^{\circ} \mathrm{C}$ pendant la première décennie, elles passent à $12,1^{\circ} \mathrm{C}$ pendant la dernière (non montré).
Pendant la période 1996-2018, les températures maximales augmentent significativement au pas de temps annuel $\left(+0,3^{\circ} \mathrm{C}\right.$ entre la dernière et la première décennie de la période) et en été et automne $\left(+0,4^{\circ} \mathrm{C}\right)$. Les températures minimales n'augmentent pas significativement sauf en août $\left(+0,6^{\circ} \mathrm{C}\right.$ entre la dernière et la première décennie). Quant aux amplitudes annuelles, elles progressent de $0,3^{\circ} \mathrm{C}$.

Ces évolutions vers un climat de plus en plus sec et chaud, notamment pendant le semestre estival, depuis les années 80-90, seront à mettre en perspective avec les résultats des modèles climatrendement établis sur les deux dernières décennies, suggérant le franchissement de seuils de sensibilité thermique ou pluviométrique de l'olivier, impactant leur rendement en fruits. Le fait que les rendements et les surfaces soient stagnants pendant la période 1996-2018 serait un autre indice du caractère critique de cette période.

\subsection{Les modèles climat/rendement en pluvial et irrigué}

Les tableaux 1 et 2 présentent les résultats de la sélection pas à pas des variables entrant dans les modèles climat-rendement, respectivement en pluvial et en irrigué. Au fur et à mesure de la sélection des variables explicatives, le $\mathrm{R}^{2}$ ajusté augmente jusqu'à atteindre 0,60 pour le rendement pluvial et 0,68 pour le rendement des oliveraies irriguées. Parallèlement, les valeurs d'erreur moyenne absolue (MAE), du critère d'information d'Akaike (AIC) et d'erreur quadratique moyenne (RMSE) diminuent progressivement. Les variables prédictives sont significatives (valeurs de $p$ inférieures à 0,05$)$.

Tableau 1. Résultat de la sélection pas à pas des variables expliquant le rendement des oliveraies pluviales. $R^{2}$ : Coefficient de détermination; RMSE : Erreur quadratique moyenne ; MAE : Erreur moyenne absolue ; AIC : Critère d'information d'Akaike ; F : valeur de Fisher ; P-value : probabilité associée à la variable par le test de Student. Result of the stepwise selection of variables explaining the yield of rainfed olive groves. $R^{2}$ : Coefficient of determination; $R M S E$ : Mean square error; MAE: Mean absolute error; AIC: Akaike's information criterion; F: Fisher's value; $P$-value: probability associated with the variable by Student's test.

\begin{tabular}{|l|c|c|c|c|c|c|c|}
\hline \multicolumn{1}{|c|}{ Sélection pas à pas } & $\mathbf{R}^{\mathbf{2}}$ & $\mathbf{R}^{\mathbf{2}}$ ajusté & RMSE & MAE & AIC & F & P-value \\
\hline Etape 1 : + Pan hydro & 0,455 & 0,429 & 9,183 & 7,239 & 171,176 & 17,561 & 0,0004 \\
\hline Etape 2 : Tmin SON a-1 & 0,561 & 0,517 & 8,448 & 6,369 & 168,215 & 12,782 & 0,0402 \\
\hline Etape 3 : + Tmin août & 0,648 & 0,593 & 7,760 & 5,912 & 165,128 & 11,668 & 0,0430 \\
\hline
\end{tabular}


Tableau 2. Comme le tableau 1, pour les rendements des oliveraies irriguées. As in Table 1, for irrigated olive yields.

\begin{tabular}{|l|c|c|c|c|c|c|c|}
\hline Sélection pas à pas & $\mathbf{R}^{\mathbf{2}}$ & $\mathbf{R}^{\mathbf{2}}$ ajusté & $\mathbf{R M S E}$ & $\mathbf{M A E}$ & $\mathbf{A I C}$ & $\mathbf{F}$ & P-value \\
\hline Etape 1 : + PSON a-1 & 0,297 & 0,264 & 13,008 & 10,220 & 187,1955 & 8,875 & 0,007 \\
\hline Etape 2 : + P mars & 0,449 & 0,394 & 11,798 & 8,904 & 183,5823 & 8,159 & 0,029 \\
\hline Etape 3 : - Tmin sept a-1 & 0,555 & 0,485 & 10,882 & 8,089 & 180,6850 & 7,896 & 0,047 \\
\hline Etape 4 : Tmax juillet & 0,676 & 0,604 & 9,540 & 6,373 & 175,3838 & 9,388 & 0,018 \\
\hline Etape 5 : + Tmin février & 0,755 & 0,684 & 8,528 & 5,891 & 170,9114 & 10,503 & 0,031 \\
\hline
\end{tabular}

Les équations des modèles sont les suivantes :

Rendement pluvial $=$ +0,06 Pan hydro - 6,1 Tmin SON n-1 +5,3 Tmin août -31,27

Rendement irrigué $+0,097$ P SON n-1+0,19 P mars -9,42 Tmin septembre $\mathrm{n}-1+7,39$ Tmax juillet $+3,48$ Tmin février $-96,97$

Avec Pan hydro, la pluviométrie de l'année hydrologique (de septembre de l'année précédant la récolte jusqu'au mois d'août de l'année de récolte) ; SON = septembre, octobre et novembre; a-1: année précédant la récolte; $P$, la pluviométrie; Tmin, la température nocturne; Tmax, la température diurne. Les rendements sont exprimés en quintaux par hectares ( $q / \mathrm{ha})$, les pluies en mm et les températures en ${ }^{\circ} \mathrm{C}$.

La validation croisée permet d'évaluer la performance des modèles qui s'avèrent performants et robustes (tableau 3). En effet, l'erreur quadratique moyenne (RMSE) et l'erreur moyenne absolue (MAE) sont relativement faibles (environ 6 $\mathrm{q} /$ ha pour l'un et l'autre modèle). De plus, les coefficients de détermination sont proches de ceux obtenus lors du calage : 0,5 pour le modèle climatrendement pluvial contre 0,6 lors du calage ; 0,6 pour le modèle climat-rendement irrigué contre 0,68 lors du calage.

Tableau 3. Résultats de la validation croisée (méthode LOOCV). Results of the cross-validation (LOOCV method).

\begin{tabular}{|l|c|c|c|c|c|}
\hline Modèles & $\begin{array}{c}\text { Nombres de } \\
\text { variables }\end{array}$ & $\mathbf{R}^{\mathbf{2}}$ & & RMSE & MAE \\
\hline Modèle climat-rendement pluvial & 3 & 0,5 & & 8,58 & 7,18 \\
\hline Modèle climat-rendement irrigué & 5 & 0,6 & & 9,52 & 7,78 \\
\hline
\end{tabular}

Dans les deux modèles, des pluies abondantes, entre 500 et $600 \mathrm{~mm}$, permettent de bons rendements. Ces chiffres sont cohérents avec ceux relevés lors des enquêtes auprès des oléiculteurs. Il faut qu'elles soient permanentes dans le cas de l'oliveraie pluviale alors qu'en irrigué, elles sont particulièrement importantes en automne pour la recharge hydrique des sols et en mars lors de la reprise de la végétation après l'hiver et pour le remplissage des réservoirs. Grâce à l'irrigation, les pluies du reste de l'année ont moins d'importance.

Dans les deux modèles, les températures nocturnes d'automne doivent rester fraîches pour limiter la respiration et favoriser la reprise de la végétation après l'été, au moment de la recharge hydrique des sols. De fortes températures d'été sont aussi un facteur favorable, y compris de jour, comme c'est le cas en irrigué ; elles permettent la maturation des fruits. Enfin, en irrigué, le gel hivernal doit être limité ; certaines années comme 2005, avec de fortes chutes de neige et un gel prononcé (jusqu'à $-20^{\circ} \mathrm{C}$ dans les montagnes et $-8,5^{\circ} \mathrm{C}$ à Jaén), ont été catastrophiques pour les oliviers dont il a fallu couper de nombreuses branches, d'après les témoignages des agriculteurs lors des ateliers.

La forte dépendance des rendements oléicoles à la pluviométrie doit s'entendre dans un contexte où cette dernière a fortement diminué depuis les années 80 , affectant particulièrement les oliveraies pluviales. Les conditions climatiques pendant la période de recharge hydrique précédant le repos hivernal sont critiques pour les rendements en pluvial et en irrigué des 20 dernières années. Alors que les températures minimales ont augmenté significativement depuis le milieu des années 80, le rendement des oliveraies des 20 dernières années est sensible à des 
températures nocturnes trop douces, qui diminuent l'efficacité de la recharge hydrique automnale. Les amplitudes thermiques diurnes, importantes dans cette région continentale et montagneuse, ayant particulièrement augmenté depuis la fin des années 80, des températures nocturnes douces sont favorables à la maturation des olives pendant l'été, évitant au fruit un trop fort contraste avec les températures diurnes très élevées. En revanche, alors que plusieurs auteurs alertent sur les seuils thermiques maximaux supportés par l'olivier (Gálan et al., 2008 ; Orlandi et al., 2020), les températures maximales n'interviennent pas dans le modèle pluvial et sont même favorables aux rendements en irrigué. Une hypothèse serait que ces températures diurnes élevées favoriseraient la maturation des fruits, tout en étant compensées par une bonne alimentation hydrique du sol par l'irrigation.
La figure 4 permet de comparer les rendements moyens simulés et observés. Les niveaux de rendements et leur forte variabilité interannuelle sont en général bien reproduits. Néanmoins, les modèles ont tendance à sur ou sous-estimer le rendement de l'olivier certaines années. En effet, les résidus peuvent être soit positifs, comme en 2003, 2007, 2018, soit négatifs comme en 2012 et 2017, en pluvial comme en irrigué. Les écarts-types des résidus sont en moyenne de 7,2 q/ha en pluvial et de 7,5 q/ha en irrigué, avec des valeurs pouvant atteindre $+11 \mathrm{q} / \mathrm{ha}$ en pluvial en 2000 et +17 q/ha en irrigué en 2011 .

Certaines années cependant, les résidus ne sont pas de même signe en pluvial et en irrigué, indiquant que des facteurs ont pu jouer favorablement pour certains vergers et à l'inverse pour d'autres.
Figure 4. Rendements observés et simulés dans la province de Jaén, dans les oliveraies irriguées et pluviales. Observed and simulated yields in the province of Jaén, in irrigated and rainfed olive groves.

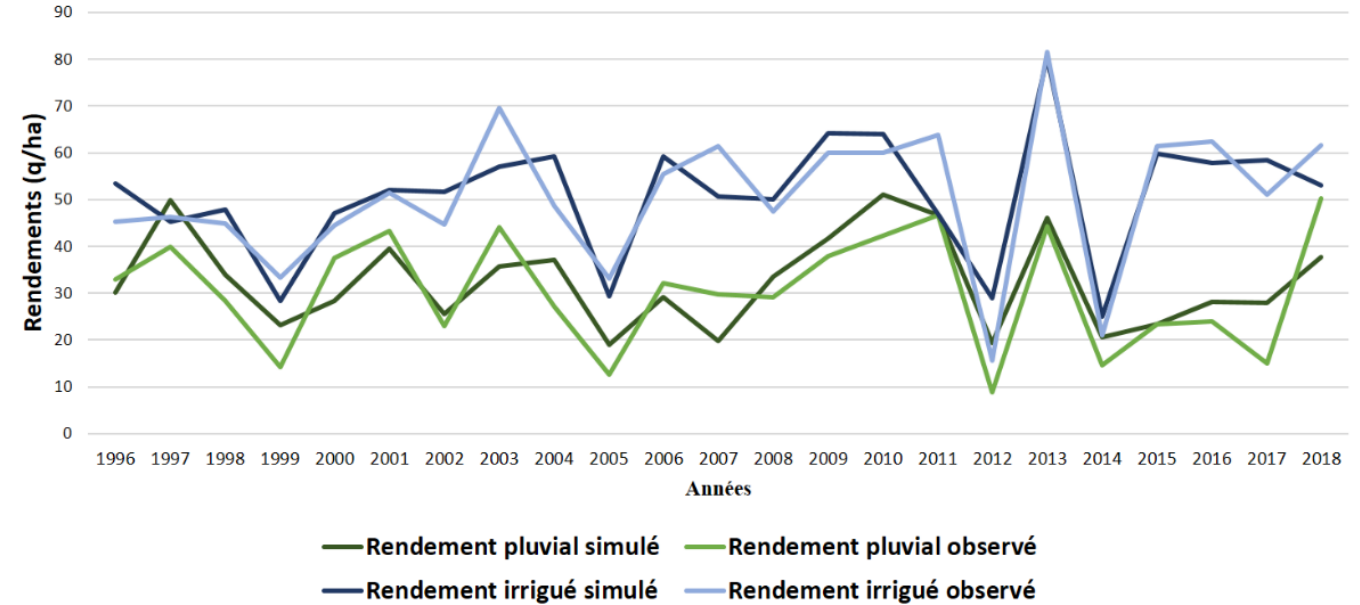

\subsection{Cartographie des rendements théoriques de l'oliveraie}

Des cartes de rendement théorique de l'oliveraie sont proposées pour l'ensemble de la province de Jaén, année après année et en moyenne pour la période 1996-2018, y compris dans les zones actuellement non plantées en oliveraie. En effet, ces cartes ayant vocation à être comparées à celles des rendements futurs afin de visualiser où les rendements resteront potentiellement bons dans le futur, il est important que tout le territoire soit cartographié. C'est pourquoi la carte de localisation des oliveraies actuelles (figure $2 \mathrm{a}$ ) doit être prise en compte lors de la lecture des cartes de rendements théoriques, car les oliveraies, même si elles sont très répandues en 2018, ne sont pas présentes dans toute la province.

Les figures 5 et 6 reflètent la supériorité des rendements des oliviers irrigués, la très forte variabilité des rendements d'une année sur l'autre, liée à l'alternance des oliviers et à la variabilité du climat, et également le fait que les années à faibles/forts rendements ne sont pas les mêmes en pluvial et en irrigué. En pluvial, l'année 2005 a le rendement le plus faible (19 q/ha en moyenne) et l'année 2010 le rendement le plus fort (51 q/ha). Par contre, 2014 a le rendement le plus faible en irrigué $(24,9 \mathrm{q} / \mathrm{ha})$ tandis que 2013 a le plus fort $(81,5 \mathrm{q} / \mathrm{ha})$. 

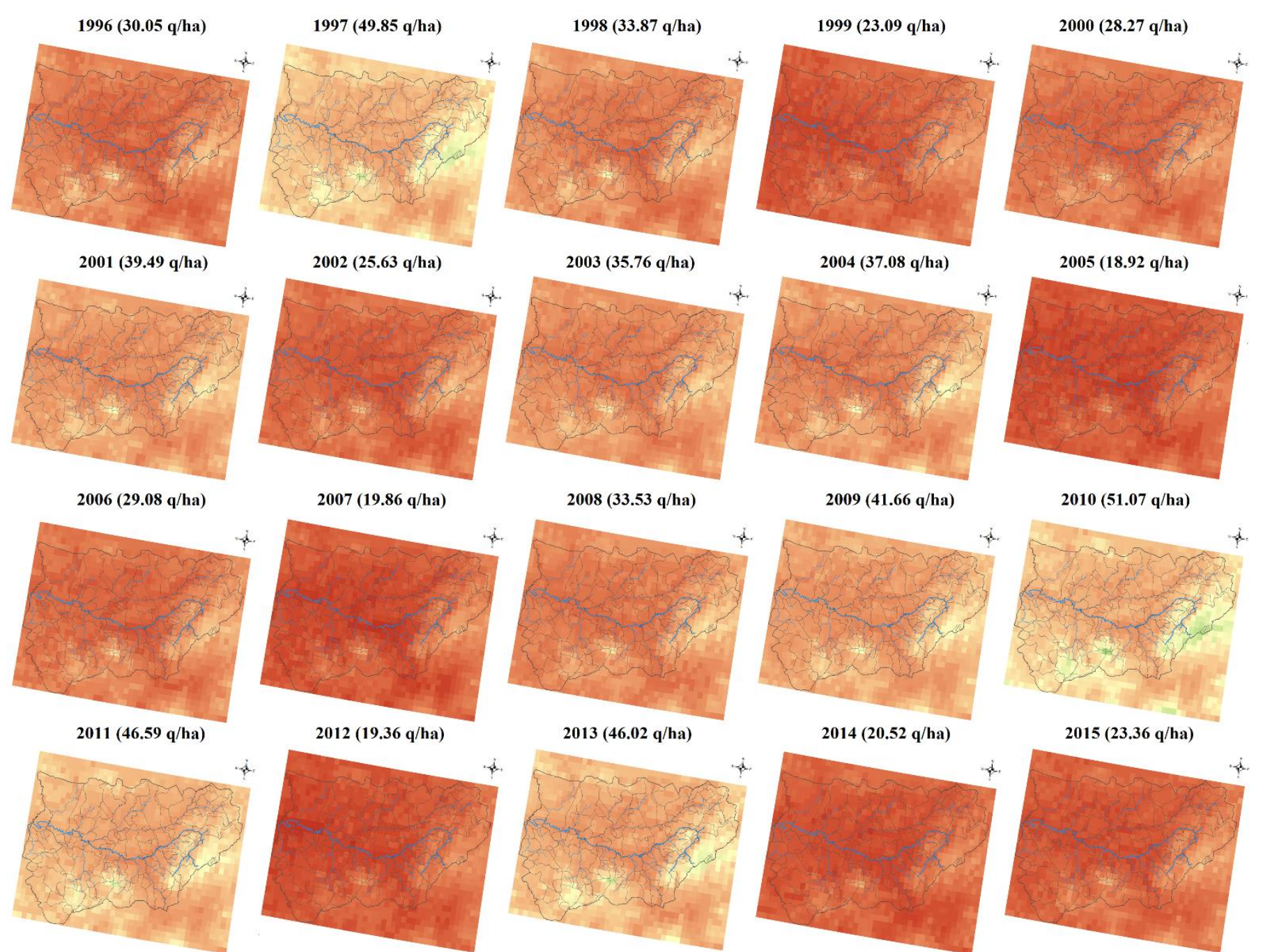

$2013(46.02 \mathrm{q} / \mathrm{ha})$
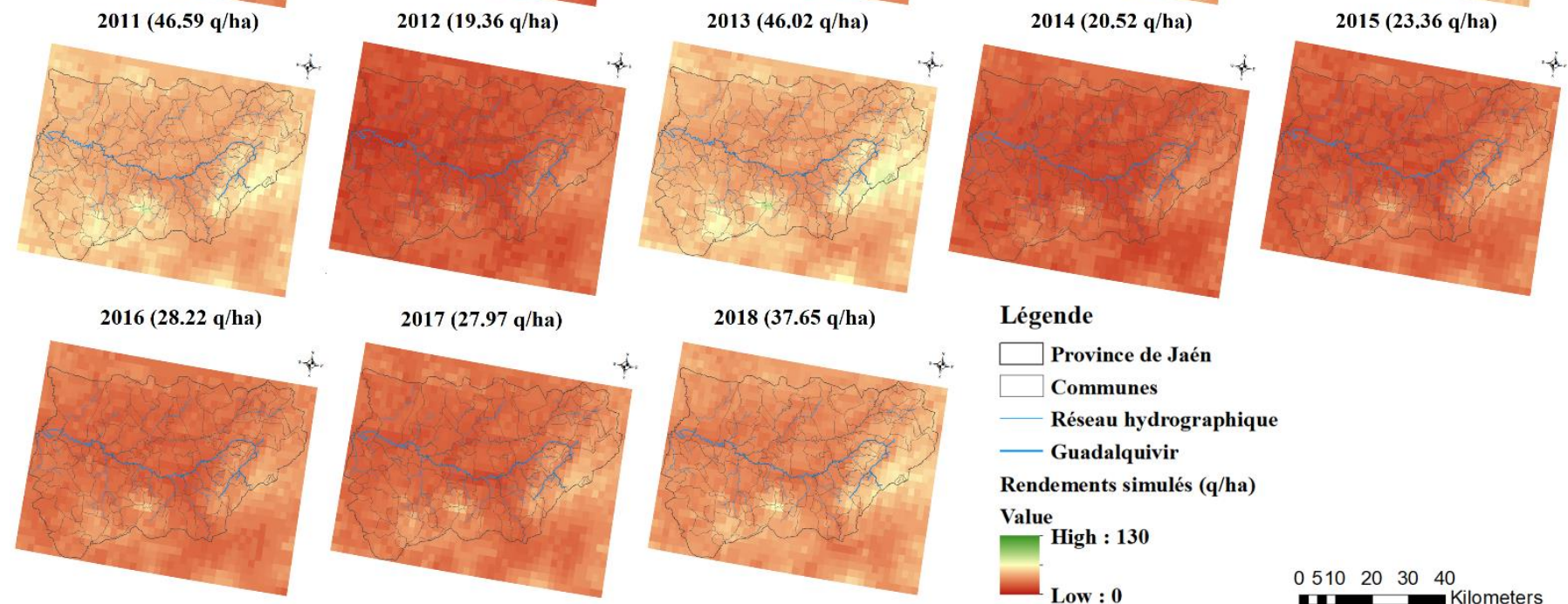

Légende

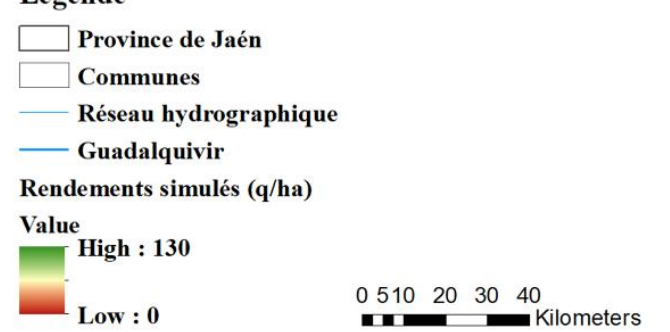

Figure 5. Spatialisation (résolution $4 \mathrm{~km}$ ) du rendement théorique des oliveraies pluviales dans la province de Jaén de 1996 à 2018. Entre parenthèses, le rendement moyen calculé sur la fenêtre, en quintaux par hectare. En bleu, le réseau hydrographique. Spatialization (4km resolution) of the theoretical yield of rainfed olive groves in the province of Jaén from 1996 to 2018. In brackets, the average yield calculated over the window, in quintals per hectare. In blue, the hydrographic network.

Les rendements théoriques les plus forts sont observés aussi bien en irrigué qu'en pluvial dans la cordillère bétique et la sierra de Cazorla au sud et sud-est, et dans la Sierra de Andujar au nord-ouest de la région, où les précipitations sont les plus abondantes (plus de $600 \mathrm{~mm}$; CNIG, 2021) et à l'exception des vallées qui les traversent, moins pluvieuses ; ce qui est cohérent avec les liens forts entre rendements et pluies. Il est notable que les régions à plus fort rendement théorique sont celles où il y a peu d'oliveraies (figure $2 \mathrm{a}$ ), sauf dans la partie sud-ouest de la province. A l'inverse, les 
zones aux plus faibles rendements théoriques se situent le long du Guadalquivir et de son affluent de rive droite, le Guadalimar, peu arrosés. Les résultats présentent donc des valeurs contrastées entre les zones de plaine et de montagne, les secteurs montagneux plus arrosés présentant un potentiel de rendement plus élevé.
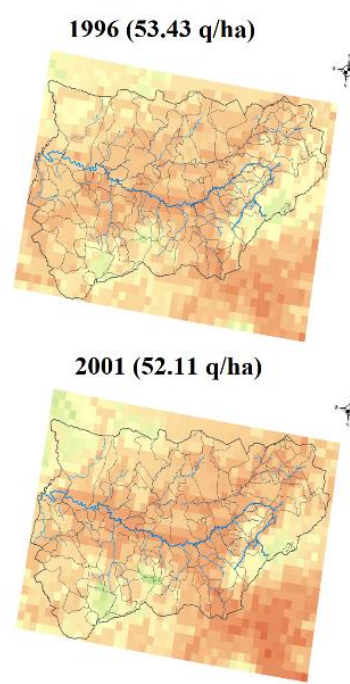

$2006(59.19$ q/ha)

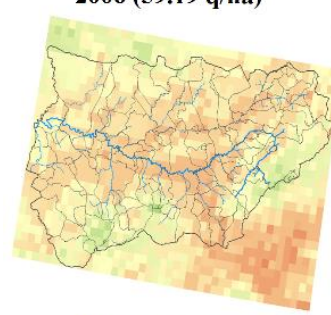

$2011(46.8$ q/ha)

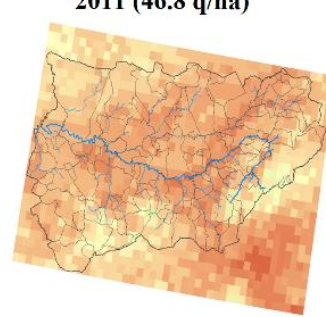

$2016(57.89$ q/ha)

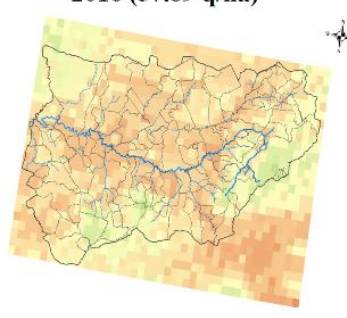

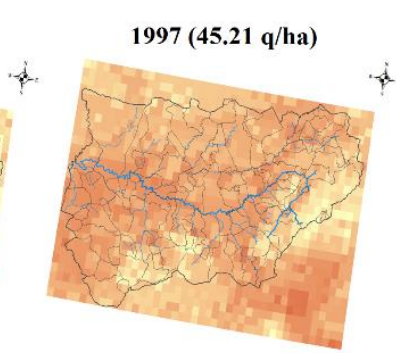

2002 (51.69 q/ha)
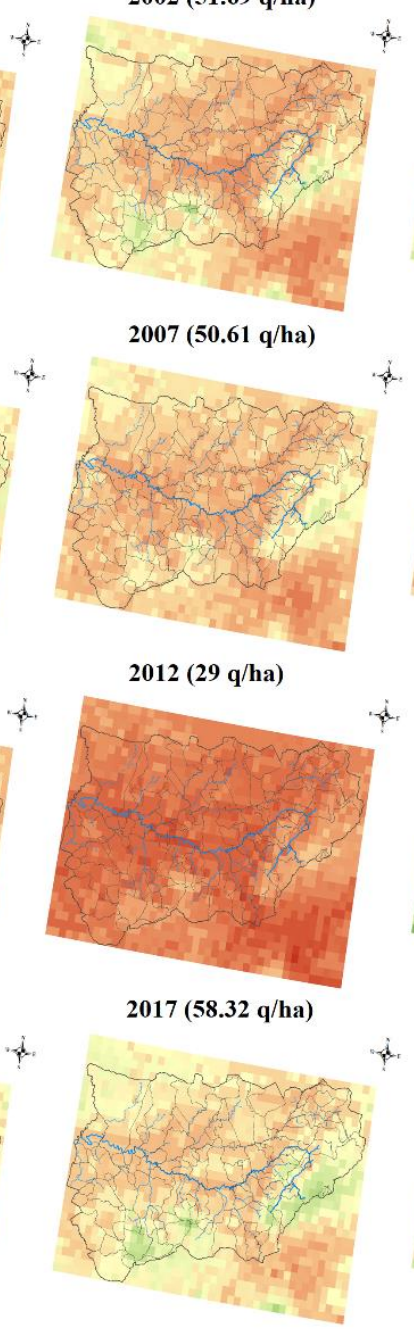

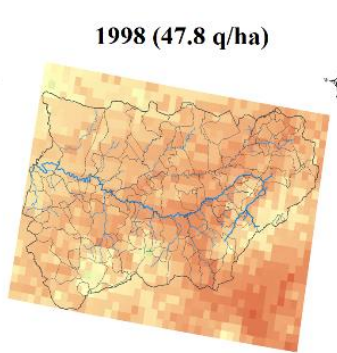

2003 (56.93 q/ha)
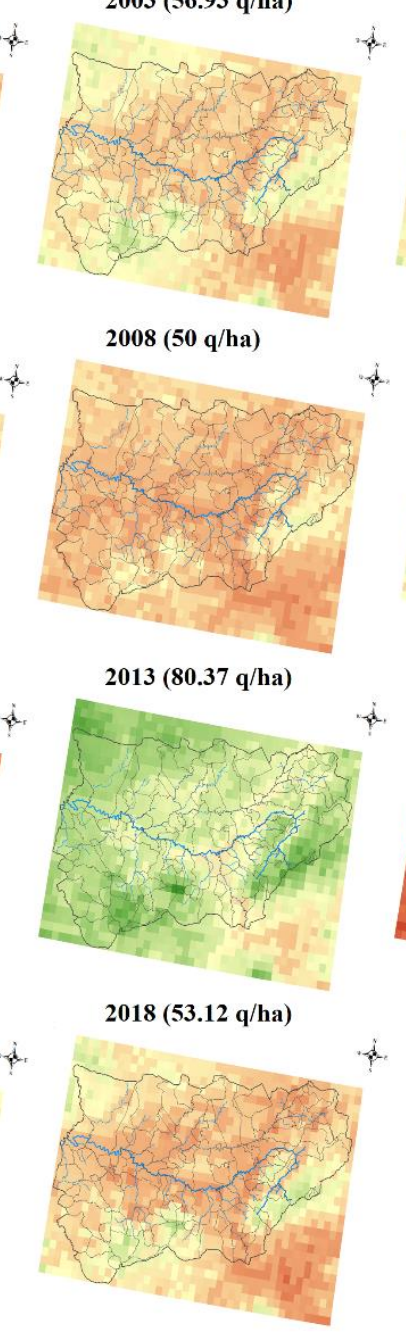
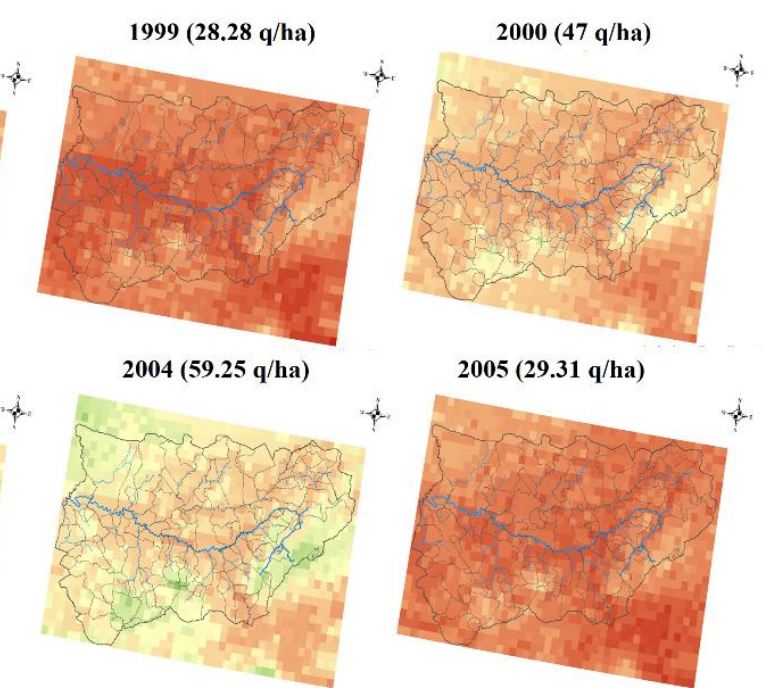

$2009(64.23$ q/ha)
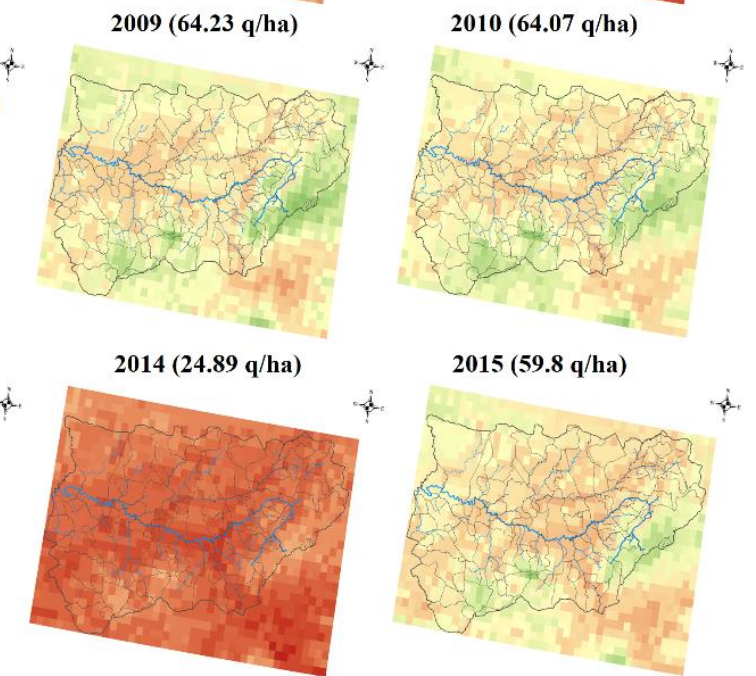

Légende

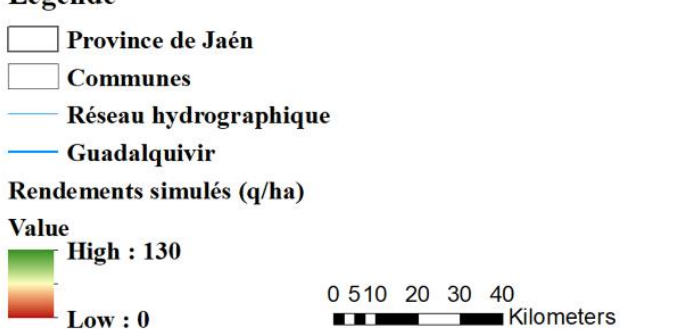

Figure 6. Même légende que pour la figure 5, pour les oliveraies irriguées. Same legend as Figure 5, for irrigated olive groves.

Les rendements moyens théoriques simulés obéissent à des répartitions spatiales similaires (figures $7 \mathrm{a}$ et $7 \mathrm{~b}$ ). Ils sont en moyenne très proches (32,5 q/ha en pluvial et 50,8 q/ha en irrigué) des valeurs observées pour l'ensemble de la province de Jaén (30,5 q/ha en pluvial et 50,6 q/ha en irrigué) (figure 7c). La variabilité des valeurs observées est toutefois plus forte que celle des valeurs simulées ; 
en pluvial, le coefficient de variation des valeurs observées est de 0,40 contre 0,31 pour les valeurs simulées ; et en irrigué, il est de 0,3 contre 0,26 . Les corrélations entre les rendements simulés et observés, en pluvial et en irrigué, sont fortes et proches $(0,8$ avec $\mathrm{p}=0,04$ et 0,87 avec $\mathrm{p}=0,03$, respectivement).
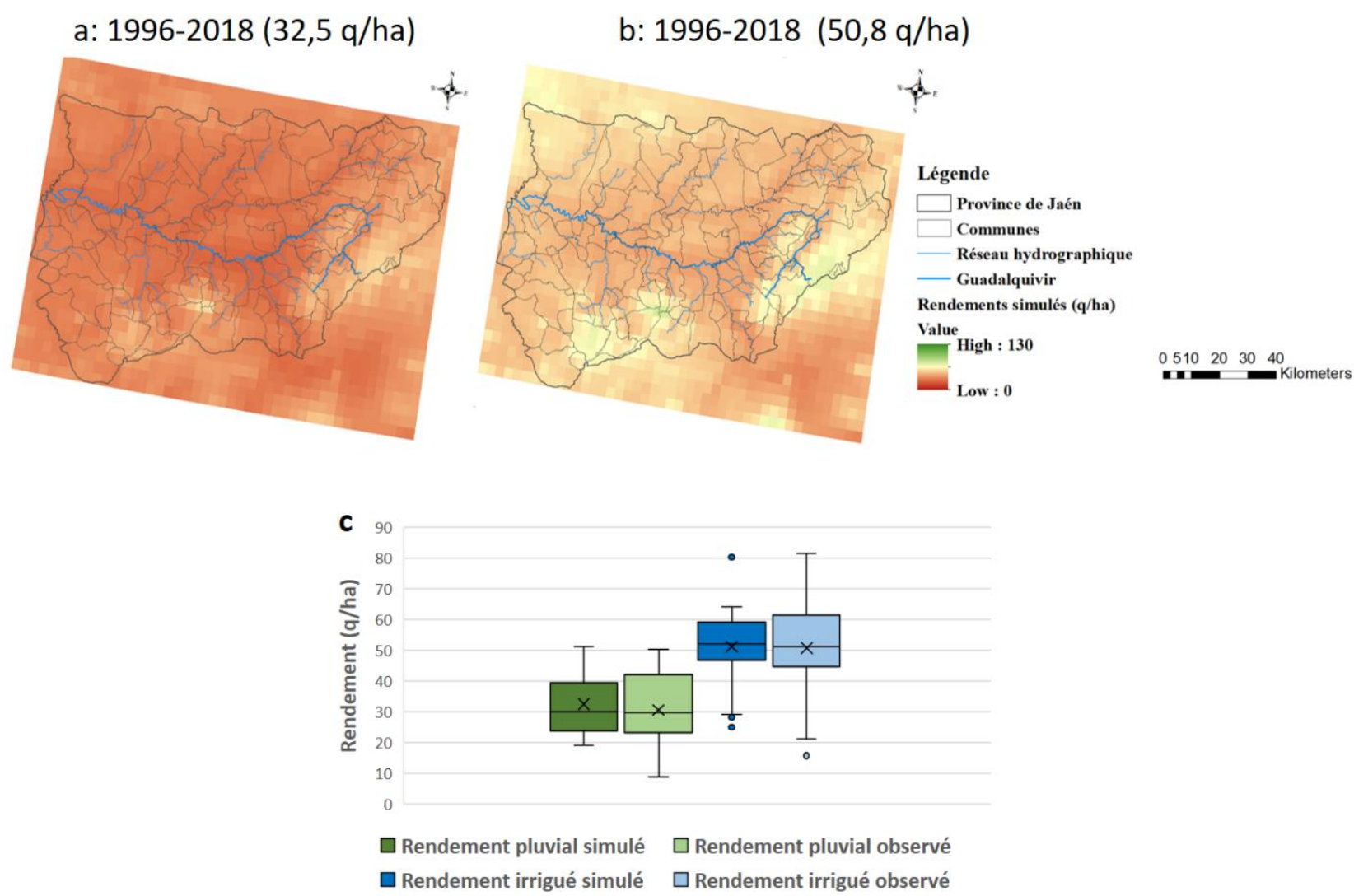

Figure 7. Rendement simulé théorique moyen (1996-2018, en q/ha), a) en pluvial et b) en irrigué dans la province de Jaén. Entre parenthèses, le rendement moyen calculé sur la fenêtre, en quintaux par hectare. c) Distribution statistique des rendements annuels des oliveraies en pluvial et en irrigué (q/ha), théoriques (simulés) et observés dans la province de Jaén. Average theoretical simulated yield (1996-2018, in q/ha), a) rainfed and b) irrigated in the province of Jaén. In brackets, the average yield calculated over the window, in quintals per hectare. c) Statistical distribution of annual yields of rainfed and irrigated olive groves ( $q / h a)$, theoretical (simulated) and observed in the province of Jaén.

\section{Discussion}

Le jeu de données TerraClimate (TC) présente l'inconvénient de ne pas fournir de données quotidiennes, donc de ne pas permettre l'introduction de variables (degrés-jours, nombre de jours de gel, nombre de jours avec une température diurne supérieure à un certain seuil) et qui se sont révélées explicatives des rendements (Badia-Canes Maquet, 2018), ont été utilisées dans les travaux sur la prédiction des dates de la phénologie des oliviers (Orlandi et al., 2005, entre autres) et qui ont été mentionnés dans les ateliers conduits avec les oléiculteurs.
La résolution spatiale de TC est moindre que celle d'autres produits, ce qui fait que son utilisation est discutable compte tenu du caractère montagneux de notre domaine. Toutefois, les produits à très haute définition utilisent des gradients orographiques qui sont très approximatifs, car les observations manquent. Prendre un produit à haute résolution spatiale sans pouvoir vérifier sa représentation orographique est un gros pari. Prendre un produit plus grossier spatialement est à notre avis plus raisonnable car il repose moins sur l'interpolation et le gradient orographique utilisés pour produire ces réanalyses. 
En revanche, les données TC présentent l'avantage 1) de fournir de bons résultats dans notre région quand on les compare aux données observées disponibles (corrélations supérieures à 0,9 entre les données observées et TC), 2) d'être actualisées annuellement et donc d'être disponibles pour les périodes très récentes, 3) d'être accompagnées de données futures pour des mondes à +2 et à $+4^{\circ} \mathrm{C}$ audessus du niveau préindustriel, ce qui est important pour la suite de notre projet, notamment la communication des résultats aux oléiculteurs.

Il n'en reste pas moins que la résolution spatiale des données TC est une limite à notre étude, de même que le manque de données annuelles, pour toutes les années de la période étudiée, de surfaces en oliveraies pluviales et irriguées et de productions et de rendements à l'échelle communale. Ces données permettraient de mieux valider notre simulation des rendements, voire d'établir des modélisations rendements-climat à échelle plus locale. La recherche de ces données fait partie de notre stratégie actuelle; elle nous permettra d'améliorer ce premier essai.

Des modèles climat-rendements sont mis en place pour les oliveraies pluviales et irriguées pour reconstituer sur des pixels de $4 \mathrm{~km}$ de côté des valeurs de rendements initialement agrégées pour la province de Jaén. Ces modèles font appel à des variables climatiques qui sont reconnues comme importantes pour les oliveraies, qui font sens et qui sont déjà mentionnées dans la bibliographie. Ces résultats sont assez similaires à ceux obtenus dans la même région par Cohen et al. (2014) et BadiaCanes Maquet (2018) en ce qu'ils mettent en avant l'importance des pluies. Cependant, les modèles antérieurs mettaient l'accent sur les pluies intégrées sur deux voire trois années, ce qui n'est plus le cas ici, peut-être parce que la période d'étude a changé et n'intègre plus la longue sécheresse des années 90 . D'autres auteurs travaillant dans la même région ou en Italie soulignent également l'importance des pluies de printemps, mais leurs modèles montrent aussi l'importance des pluies d'été (Galán et al., 2008 ; Quiroga et Iglesias, 2009 ; Orlandi et al., 2013 ; Rodrigo-Comino et al., 2021) ou d'hiver (Aguilera et Ruiz-Valenzuela, 2019). Enfin, ces modèles améliorent les performances de ceux de
Cohen et al. (2014) grâce à l'introduction de variables caractérisant la température. Les résultats concernant les températures sont cohérents avec ceux de Galán et al. (2008), Aguilera et RuizValenzuela (2019) et Arenas-Castro et al. (2020) qui, pour les oliveraies andalouses, insistent sur l'importance des températures maximales et minimales d'été et d'automne pour les premiers auteurs, et sur celles d'hiver pour les seconds et le troisième.

Ces modèles sont statistiquement robustes et performants; il y a une bonne adéquation entre valeurs observées et simulées. Les résidus peuvent être liés à des facteurs climatiques non pris en compte par les modèles (figure 4). Par exemple, en pluvial, des pluies abondantes lors de la récolte en automne $(1999,2012)$ peuvent faire chuter les olives ou au contraire, des pluies insuffisantes à l'automne peuvent les assécher et les faire flétrir (2004 et 2017). Les facteurs climatiques peuvent aussi avoir une influence sur les conditions de la récolte, par exemple des pluies excessives en automne peuvent la compliquer, beaucoup de parcelles étant desservies par des chemins non goudronnés.

Des modèles plus complexes permettraient peutêtre d'améliorer la capacité de prédiction des rendements. Pour cela, il serait essentiel de prendre en compte de nouvelles variables dans les modèles telles que celles mentionnées ci-dessus et en suivant les indications qui sont apparues lors d'ateliers avec les agriculteurs (fréquence du gel, occurrence de vent de sud desséchant, longues séquences chaudes d'été, ...). Il faudrait pour cela recourir non plus à des valeurs mensuelles mais à des valeurs quotidiennes. Des variables biologiques mesurant la présence de ravageurs et de maladies pourraient compléter l'explication des rendements; elles pourraient être relatives à des bactéries, des ravageurs (e.g. mouche de l'olive, teigne de l'olivier et la cochenille noire), des maladies (e.g. l'œil de paon, verticilliose, brunissement). La mouche de l'olive (Bactrocera oleae) est le principal ravageur de l'olivier. Les dégâts occasionnés par la larve peuvent remettre en cause la totalité de la récolte (Ponti et al., 2014). 
Si la validation temporelle des simulations donne des résultats honorables (section 2.3), une tentative de réaliser une validation spatiale a été moins fructueuse. Les données simulées à l'échelle des pixels climatiques ont été confrontées aux données de rendements disponibles à échelle communale en 1996 et 1997. Les données communales ne séparant pas les valeurs des vergers irrigués et non irrigués, les rendements théoriques calculés ont été pondérés par la proportion des deux types de vergers à l'échelle de la province en 1998, alors que le taux d'oliveraies irriguées est variable selon les communes. Les données de rendements théoriques calculées pixel par pixel ont été moyennées par commune et comparées aux observations. Les résultats ne sont pas concluants. Les rendements simulés sont en général supérieurs aux rendements observés et les répartitions spatiales des rendements observés et simulés ne sont pas les mêmes.

Pour améliorer cette validation, il faudrait prendre en compte la proportion de surfaces irriguées dans chaque commune pendant les deux années durant lesquelles des statistiques communales sont disponibles. Les cartes générées à partir du site Géoportal du Ministère MAGRAMA montrent en effet un changement majeur entre 1980-1990 et 2000-2010 (sig.mapama.gob.es/siga/), de même que les cartes des surfaces irriguées en 1982 et 1999 établies par Jiménez et al. (2002). L’irrigation, limitée en 1982 aux abords immédiats des grandes vallées du Guadalquivir et du Genil et générant des rendements assez élevés s'est étendue aux collines environnantes en 1999 suite à la grande sécheresse des années 1993 à 1995, induisant une augmentation du rendement moyen.

Toutefois, il existe des divergences importantes entre les sources concernant les surfaces irriguées. Par exemple, en 1999, elles représentent $1091 \mathrm{~km}^{2}$ selon les statistiques du MAGRAMA et $1579 \mathrm{~km}^{2}$ selon Jiménez et al. (2002). Ce différentiel s'explique par le fait que la mise en œuvre de l'irrigation est préalable à l'officialisation par l'organisme gestionnaire du bassin (Confédération Hydrographique du Guadalquivir - CHG), qui peut intervenir plusieurs années plus tard (c.o. J.D. Sánchez-Martínez, enquêtes de terrain, 2018). Or, les statistiques officielles ne comptent parmi les oliveraies irriguées que celles dont le statut a été officialisé.

Les modèles climat-rendement générés à l'échelle de la province de Jaén intègrent donc temporellement et spatialement les changements d'occupation des sols qui se sont produits dans les communes. Ces changements pourraient expliquer le fait que les rendements observés soient plus faibles que les rendements simulés, car certains vergers sont encore jeunes et peu productifs à la fin des années 90 et leur croissance n'a pas encore eu d'effet sur les rendements. Les modèles climatrendement n'intègrent pas non plus la variabilité spatiale de l'occupation du sol, notamment les différences topographiques très notables à l'échelle communale.

Les cartographies des rendements théoriques actuels ont vocation à être comparées avec celles des rendements potentiels futurs, ce qui permettra de montrer les zones où les pertes de rendements seront les plus importantes, à techniques de culture égales. C'est ce qui sera effectué dans un travail futur. En effet, l'adaptabilité des systèmes agricoles au changement climatique dans les régions au climat irrégulier et sec, comme le bassin méditerranéen, est essentielle pour la durabilité et la viabilité de l'activité oléicole de nombreuses zones rurales. Elle dépend des choix des sociétés locales, des modèles agricoles promus par les politiques publiques, des marchés internationaux (CCAFS, 2009 ; World Bank, 2010 ; O'Brien et al., 2011) et d'une organisation au niveau communautaire (Heltberg et al., 2009).

Malgré les avantages des techniques d'adaptation à court et à long termes, l'application d'un système d'irrigation entraînera des coûts supplémentaires d'infrastructure et d'énergie pour les producteurs cultivant l'olivier pluvial. De plus, la demande en eau d'irrigation et les conflits d'usage augmenteront dans le futur (Tanasijevic et al., 2014) alors que les ressources en eau diminueront (Garrote et al., 2015 ; Donnelly et al., 2017). Ceci limitera la possibilité d'une extension des oliveraies irriguées, voire questionnera la viabilité des oliveraies irriguées existantes. Certains agriculteurs considèrent toutefois qu'une dotation 
de $1000 \mathrm{~m}^{3} / \mathrm{ha} /$ an serait suffisante pour obtenir des rendements corrects, alors qu'à présent, elle est de $1500 \mathrm{~m}^{3}$ et n'est pas dépensée intégralement chaque année (source: données d'enquêtes, 2018). La diversification variétale pourrait également réduire les besoins en eau d'irrigation, en introduisant peu à peu dans les vergers des variétés cultivées dans des climats plus secs (ex. Tunisie), en particulier dans les vergers de plaine et les vergers pluviaux.

On peut supposer que la délocalisation des oliveraies dans les régions montagneuses, mieux arrosées et plus fraîches, pourrait être très bénéfique pour le secteur oléicole de la province de Jaén dans le futur, lorsque le climat deviendra plus sec et plus chaud, comme prévu pour l'avenir (MedECC, 2020). La délocalisation en montagne semble une bonne solution sur le papier, mais il y a plusieurs obstacles : les risques de dégradation des ressources en eau liés à une déforestation ne sont pas négligeables, de même que les difficultés posées par les pentes fortes et par le classement en parc naturel pour le défrichement et la mise en culture. A cela s'ajoute pour des oliveraies irriguées, le coût de la mise en place d'une infrastructure d'irrigation alors que le réseau hydrographique est moins dense et moins aménagé en montagne. Les cartes seront des vecteurs de communication auprès des agriculteurs, car nos résultats suggèrent des choix difficiles qui doivent être anticipés.

\section{Conclusion}

L'objectif de ce travail était de mettre au point des méthodologies statistiques et cartographiques pour affiner la connaissance spatiale des rendements des oliveraies pluviales et irriguées de la province de Jaén (Andalousie) qui ne sont disponibles qu'à l'échelle provinciale. Par la suite, la simulation des rendements potentiels futurs toujours à échelle relativement fine, dans un contexte plus chaud et plus sec comme attendu dans le bassin méditerranéen, permettra de montrer aux agriculteurs les zones où les pertes de rendement seront les plus importantes, à techniques de culture égales.

Dans un premier temps, l'analyse des rendements dans la province de Jaén (1959-2018) montre qu'ils augmentent considérablement entre la fin des années cinquante et 1996, puis stagnent autour de 30,5 et $50,6 \mathrm{q} / \mathrm{ha}$ pour les vergers pluviaux et irrigués, respectivement (moyenne 1996-2018). Pendant cette même période, les pluies sont de $11 \%$ inférieures à ce qu'elles étaient avant 1979, année de rupture significative dans la série pluviométrique et les températures maximales (minimales) annuelles sont de $1,4^{\circ} \mathrm{C}(0,9)$ supérieures à celles de la première décennie de la période d'étude (19591968). Les plus fortes élévations de températures sont observées pendant la saison chaude. C'est donc dans ce contexte dégradé que doivent être interprétés les résultats de l'étude.

Ensuite, des modèles climat-rendement, en pluvial et en irrigué, sont élaborés à partir de données de rendements provinciales et de données de précipitations et de températures à haute résolution (TerraClimate, pixels de $4 \mathrm{~km}$ ), moyennées sur les zones en oliveraies de la province de Jaén. Cette modélisation s'effectue sur la période de rendements stationnaires 1996-2018. La sélection stepwise des variables explicatives a permis d'obtenir des modèles performants et la procédure leave-one-out cross-validation (LOOCV) de les valider. Les variables climatiques, en premier lieu les pluies, puis les températures d'automne et enfin celles d'été et de fin d'hiver, expliquent $60 \%$ de la variabilité des rendements en pluvial et $68 \%$ de celle des rendements en irrigué.

Enfin, des rendements théoriques moyens actuels (1996-2018) sont calculés sur des pixels de $4 \mathrm{~km}$ à partir des équations liant rendements et variables climatiques. Leur cartographie montre que les parcelles irriguées ont un rendement de 18 q/ha supérieur à celui des parcelles en pluvial, ce qui est très proche des valeurs observées. Les cartographies indiquent également des rendements souvent plus forts là où il n'y a pas d'oliveraie, mais où la pluviométrie est importante (contraste plaine/montagne). Par conséquent, il est possible d'anticiper qu'un éventuel déplacement des oliveraies dans les régions montagneuses pourrait être très bénéfique pour le secteur oléicole de la province de Jaén dans le futur, lorsque le climat deviendra encore plus sec et plus chaud. Une autre option consisterait à remplacer peu à peu les oliviers 
de la variété Picual, actuellement dominante dans la région, par une variété plus résistante à la sécheresse, dans les oliveraies de plaine. L'adoption de certaines mesures d'adaptation à court et à long terme peut être cruciale, même si leur application peut également entraîner des coûts socioéconomiques importants (Fraga et al., 2021 ; Cabezas et al., 2021), qui pourraient être partiellement compensés par une meilleure valorisation économique de l'huile d'olive, encore largement vendue en vrac, sans le bénéfice du label de l'Indication Géographique Protégée (IGP).

Remerciements : les auteurs remercient l'Institut de la Transition Environnementale de Sorbonne Université qui finance leur recherche. Les auteurs remercient également les chercheurs et étudiants de master ayant participé aux enquêtes (Salvador Gil, Thalis Gropetit, Yelli El Arch, Marta Martinez) et l'association PASOS qui anime les ateliers participatifs avec les oléiculteurs.

\section{Références}

Abatzoglou J. T., Dobrowski S. Z., Parks S. A., Hegewisch K. C., 2018. TerraClimate, a high-resolution global dataset of monthly climate and climatic water balance from 1958-2015. Scientific data, 5, 1-12. 10.1038/sdata.2017.191fotosymapas.wordpress.

Aguilera F., Ruiz-Valenzuela L., 2019. A new aerobiological indicator to optimize the prediction of the olive crop yield in intensive farming areas of southern Spain. Agricultural and Forest Meteorology, 271, 207-213.

Akaike H., 1974. A new look at the statistical model identification. IEEE transactions on automatic control, 19, 716-723. 10.1109/TAC.1974.1100705.

Angles S., 2016. Oléiculture, systèmes oléicoles et territoires méditerranéens : de la filière au paysage. Thèse de Doctorat, Université Paris 13, 165 p.

Araque Jiménez E., Gallego Simón V. J., Sánchez-Martínez J. D., 2002. El olivar regado en la provincia de Jaén. Investigaciones geográficas. Universidad de Alicante, 28, 532.

Arenas-Castro S., Gonçalves J., Moreno M., Villar R., 2020. Projected climate changes are expected to decrease the suitability and production of olive varieties in southern Spain. Science of the Total Environment, 709, 136161.

Badia-Canes Maquet, J., 2018. Modélisation du rendement des oliveraies dans la province de Jaén, Espagne. Mémoire de M1 - Hydrologie, Hydrogéologie, Géochimie environnementale, Sorbonne Université, Laboratoires Métis et Locean, 33 p.
Baldy C., 1990. Le climat de l'Olivier (Olea europaea L.). Ecologia mediterranea, 16, 113-121.

Cabezas J. M., Ruiz-Ramos M., Soriano M. A., Santos C., Gabaldón-Leal C., Lorite I. J., 2021. Impact of climate change on economic components of Mediterranean olive orchards. Agricultural Water Management, 248, 106760.

CCAFS, 2009. Climate change, agriculture and food security: A CGIAR challenge program. Alliance of the CGIAR Centers and ESSPR, ep. 1, 51 pp. [Available online at http://r4d.dfid.gov.uk/PDF/Outputs/CCAFS/ccafs_report_1_1 owresolution.pdf].

Centre National de l'Information géographique (CNIG), 2021. centrodedescargas.cnig.es/CentroDescargas/busquedaRedirigi da.do?ruta=PUBLICACION_CNIG_DATOS_VARIOS/Mapa sGenerales/Mapa-fisico-de-Espana_mudo-1-3.000.000.pdf

Cohen M., Ronchail J., Alonso-Roldán M., Morcel C., Angles, S., Araque-Jiménez E., Labat D., 2014. Adaptability of Mediterranean agricultural systems to climate change. The example of the Sierra Mágina olive-growing region (Andalusia, Spain). Part I: past and present. Weather, Climate, and Society, 6, 380-398, 10.1175/WCAS-D-12-00043.1.

Corine Land Cover (CLC), 2021, données d'occupation des sols, land.copernicus.eu/pan-european/corine-land-cover

Donnelly C., Greuell W., Andersson J., Gerten D., Pisacane G., Roudier P., Ludwig F. 2017. Impacts of climate change on European hydrology at 1.5, 2 and 3 degrees mean global warming above preindustrial level. Clim. Chang., 143, 13-26.

Fick S. E., Hijmans R. J., 2017. WorldClim 2: new 1-km spatial resolution climate surfaces for global land areas. Int. J. Climatol.. 37, 4302-4315. doi.org/10.1002/joc

Fraga H., Moriondo M., Leolini L., Santos J. A., 2021. Mediterranean olive orchards under climate change: A review of future impacts and adaptation strategies. Agronomy, 11, 56. doi.org/10.3390/agronomy11010056

Gabaldón-Leal C., Ruiz-Ramos M., de la Rosa R., León L., Belaj A., Rodríguez A., Santos C., Lorite I. J., 2017. Impact of changes in mean and extreme temperatures caused by climate change on olive flowering in southern Spain. Int. J. Climatol., 37, 940-957. 10.1002/joc.5048

Galán C., García-Mozo H., Vázquez L., Ruiz L., De La Guardia C. D., Domínguez-Vilches E., 2008. Modeling olive crop yield in Andalusia, Spain. Agronomy Journal, 100, 98104, 10.2134/agrojn12006.0345.

Garrote L., Iglesias A., Granados A., Mediero L., MartinCarrasco F., 2015. Quantitative assessment of climate change vulnerability of irrigation demands in Mediterranean Europe. Water Res. Manag., 29, 325-338.

GeoPortal du MAPAMA (Ministère de l'Agriculture, de la Pêche et de l'Alimentation), 2021. Mapas de Cultivos y Aprovechamientos: Mapa de Cultivos 1980-1990 y Mapa de Cultivos 2000-2010. sig.mapama.gob.es/geoportal/.

Harris I., Osborn T. J., Jones P., Lister D., 2020. Version 4 of 
the CRU TS monthly high-resolution gridded multivariate climate dataset. Scientific Data, 7(1), 109. doi.org/10.1038/s41597020-0453-3.

Heltberg R., Bennett-Siegel P., Lau-Jorgensen S., 2009. Addressing human vulnerability to climate change: Toward a 'no regrets' approach. Global Environ. Change, 19, 89-99, 10.1016/j.gloenvcha.2008.11.003.

Hijmans R. J., Cameron S. E., Parra J. L., Jones P. G., Jarvis A., 2005. Very high resolution interpolated climate surfaces for global land areas. Int. J. Climatol.. 25, 1965-1978. doi.org/10.1002/joc. 1276.

IPCC Intergovernmental Panel on Climate Change, 2014. Climate Change 2013: The Physical Science Basis. In: Stocker T.F., Qin D., Plattner G.-K., Tignor M., Allen S.K., Boschung J., Nauels A., Xia Y., Bex V. and Midgley P.M. (Eds.), Contribution of Working Group I to the Fifth Assessment Report of the Intergovernmental Panel on Climate Change. Cambridge; New York, NY: Cambridge University Press. 1535 pp.

Jiménez E. A., Gallego Simón V. J., Sánchez-Martínez J. D., 2002. El Olivar Regado en la provincia de Jaén. Instituto Universitario de Geografía Universidad De Alicante, Investigaciones Geográfica, 28, 5-32.

Kobayashi S., Ota Y., Charada Y., Ebita A., Moriya M., Onoda H., Onogi K., Kamahori H., Kobayashi C., Endo H., Miyaoka K., Takahashi K., 2015. The JRA-55 Reanalysis: General specifications and basic characteristics. J. Meteorol. Soc. Jpn., Ser II 93, 5-48, doi.org/10.2151/jmsj.2015.

Lionello P., Scarascia L., 2018. The relation between climate change in the Mediterranean region and global warming. Regional Environmental Change, 18, 1481-1493 doi.org/10.1007/s10113018-1290-1.

Lorenzo-Lacruz J., Morán-Tejeda E., Vicente-Serrano S. M., López-Moreno J. I., 2013. Streamflow droughts in the Iberian Peninsula between 1945 and 2005: spatial and temporal patterns. Hydrology and Earth System Sciences, 17, 119-134.

MedECC, 2020. Résumé à l'intention des décideurs. Dans : Changement climatique et environnemental dans le bassin méditerranéen - Situation actuelle et risques pour le futur. Premier rapport d'évaluation sur la Méditerranée [Cramer W., Guiot J., Marini K. (eds.)] Union pour la Méditerranée, Plan Bleu, UNEP/MAP, Marseille, France, 35 pp.

Ministère de l'Agriculture, de la Pêche et de l'Alimentation (MAPA), 2021a, www.mapa.gob.es/es/agricultura/temas/produccionesagricolas/aceite-oliva-y-aceituna-mesa/aceite.aspx.

Ministère de l'Agriculture des Pêches et de l'Alimentation (MAPA), 2021b. Données de rendements, www.mapa.gob.es/es/estadistica/temas/publicaciones/anuario -de-estadistica/

Moriondo M., Ferrise R., Trombi G., Lorenzo B., Dibari C., Bindi M., 2015. Modelling olive trees and grapevines in a changing climate, Environmental Modelling \& Software, dx.doi.org/10.1016/j.envsoft.2014.12.016.
Mosier T. M., Hill D. F., Sharp K. V., 2014. 30-Arcsecond monthly climate surfaces with global land coverage. Int. J. Climatol., 34, 2175-2188. doi.org/10.1002/joc.3829.

O’Brien G., Devisscher T., O'Keefe P., Tellam I., 2011. The adaptation continuum: Groundwork for the future. Lambert Academic Publishing, 364 pp.

Oficina Comarcal Agraria (OCA), 2001. Caracterización del territorio de la OCA. Junta de Andalucia, Consejeria de Agricultura Pesca y Desarrollo Sostenible. www.juntadeandalucia.es/organismos/agriculturaganaderiapesca ydesarrollosostenible/servicios/estadistica-cartografia/estudiosinformes/detalle/95149.html.

Orlandi F., Vazquez L. M., Ruga L., Bonofiglio T., Fornaciari, M., Garcia-Mozo H., Domínguez E., Romano B., Galan C., 2005. Bioclimatic Requirements for olive flowering in two Mediterranean regions located at the same latitude (Andalucía, Spain and Sicily, Italy). Ann. Agric. Environ. Med., 12, 47-52.

Orlandi F., Avolio E., Bonofiglio T., Federico S., Romano B., Fornaciari M., 2013. Potential shifts in olive flowering according to climate variations in Southern Italy. Meteorological Applications, 20, 497-503. dx.doi.org/10.1002/met.1318.

Orlandi F., Rojo J., Picornell A., Oteros J., Pérez-Badia R., Fornaciari M., 2020. Impact of climate change on olive crop production in Italy. Atmosphere, 11, 595. 10.3390/atmos 11060595.

Ponti L., Gutierrez A. P., Ruti P. M., Dell'Aquila A., 2014. Fine-scale ecological and economic assessment of climate change on olive in the Mediterranean Basin reveals winners and losers. Proceedings of the National Academy of Sciences USA, 111, 5598-5603.

Programme des Nations Unies pour l'Environnement/Plan d'Action pour la Méditerranée PNUE/PAM et Plan Bleu, 2020. État de l'Environnement et du Développement en Méditerranée. Nairobi, planbleu.org/wp-content/uploads/2021/04/RED-2020Rapport-complet.pdf

Quénol H., Bonnardot V., 2014. A multi-scale climatic analysis of viticultural terroirs in the context of climate change: the "TERADCLIM" project. International Journal of Vine and Wine Sciences, 23-32.

Quiroga S., Iglesias A., 2009. A comparison of the climate risks of cereal, citrus, grapevine and olive production in Spain. Agricultural Systems, 101,91-100. dx. doi.org/10.1016/j.agsy.2009.03.006.

Ranasinghe R., A. C. Ruane, R. Vautard, N. Arnell, E. Coppola, F. A. Cruz, S. Dessai, A. S. Islam, M. Rahimi, D. Ruiz Carrascal, J. Sillmann, M. B. Sylla, C. Tebaldi, W. Wang, R. Zaaboul, 2021. Climate Change Information for Regional Impact and for Risk Assessment. In: Climate Change 2021: The Physical Science Basis. Contribution of Working Group I to the Sixth Assessment Report of the Intergovernmental Panel on Climate Change [MassonDelmotte V., P. Zhai, A. Pirani, S. L. Connors, C. Péan, S. Berger, N. Caud, Y. Chen, L. Goldfarb, M. I. Gomis, M. Huang, K. Leitzell, E. Lonnoy, J. B. R. Matthews, T. K. Maycock, T. Waterfield, O. Yelekçi, R. Yu, B. Zhou (eds.)]. Cambridge University Press. In Press 
Rodrigo-Comino J., Senciales-González J. M., Yu Y., Salvati L., Giménez-Morera A., Cerdà A., 2021. Long-term changes in rainfed olive production, rainfall and farmer's income in Bailén (Jaén, Spain). Euro-Mediterranean Journal for Environmental Integration, 6, 1-15, doi.org/10.1007/s41207021-00268-1.

Rodríguez-Cohard J. C., Sánchez-Martínez J. D., GallegoSimón V. J., 2017. The upgrading strategy of olive oil producers in Southern Spain: origin, development and constraints. Rural Society, 26, 30-47, 10.1080/10371656.2017.1285470.

Ronchail J., Cohen M., Alonso-Roldan M., Garcin H., Angles S., Sultan B., 2014. Adaptability of Mediterranean agro systems to climate change, 2014. The example of the Sierra Mágina olive growing region (Andalusia, Spain). II The future. Weather, Climate and Society, 6, 451-467. 10.1175/WCAS-D-12-00045.1

Sánchez-Martínez J. D., Paniza-Cabrera A., 2015. The olive monoculture in the south of Spain. European Journal of Geography, 6, 16-29.

Sánchez-Martínez J. D., Garrido-Almonacid A. G., 2017. Productivism and post-productivism in the olive groves of southern Spain. Quaestiones Geographicae, 36, 57-69.

Sánchez-Martínez J. D., Rodríguez-Cohard J. C., GarridoAlmonacid A., Gallego-Simón V. J., 2020. Social innovation in rural areas? The case of Andalusian olive oil co-operatives. Sustainability, 12, 10019, 10.3390/su122310019
Sofo A., Manfreda S., Fiorentino M., Dichio B., Xiloyannis C., 2008. The olive tree: a paradigm for drought tolerance in Mediterranean climates. Hydrology and Earth System Sciences, 12, 293-301.

Tanasijevic L., Todorovic M., Pereira L. S., Pizzigalli C., Lionello P., 2014. Impacts of climate change on olive crop evapotranspiration and irrigation requirements in the Mediterranean region. Agricultural Water Management, 144, 54-68.

Tissot C., Quenol H., Rouan M., 2020. Adaptation de la viticulture argentine à la variabilité climatique : une approche par simulation dans la région de Mendoza. Norois, 254, 91108 .

van Leeuwen C., Destrac-Irvine A., Dubernet M., Duchêne E., Gowdy M., Marguerit E., Pieri P., Parker A., de Rességuier L., Ollat N., 2019. An update on the impact of climate change in viticulture and potential adaptations. Agronomy, 9, 514, 10.3390/agronomy9090514.

World Bank, 2010. WDR, 2010: Development and Climate Change. World Bank, 417 pp. [Available online at http://wdronline.worldbank.org/worldbank/a/c.html/world_develo pment_report_2010/abstract/WB.978-0-8213-7987-5.abstract.]

Willmott C. J., Robeson S. M., 1995. Climatologically aided interpolation (CAI) of terrestrial air temperature. Int. J. Climatol.. 15, 221-229. doi.org/10.1002/joc.3370150207

Citation de l'article : Arfaoui F. et al., 2021. Évolution, modélisation et cartographie des rendements de l'oliveraie dans la province de Jaen en Espagne (1959-2018). Climatologie, 18, 4. 